\title{
Luminescent Colloidal InSb Quantum Dots from In Situ Generated Single-Source Precursor
}

\author{
Serena Busatto, Mariska de Ruiter, Johann T. B. H. Jastrzebski, Wiebke Albrecht, Valerio Pinchetti, \\ Sergio Brovelli, Sara Bals, Marc-Etienne Moret, and Celso de Mello Donega*
}

Cite This: ACS Nano 2020, 14, 13146-13160

ABSTRACT: Despite recent advances, the synthesis of colloidal InSb quantum dots (QDs) remains underdeveloped, mostly due to the lack of suitable precursors. In this work, we use Lewis acid-base interactions between $\mathrm{Sb}$ (III) and In(III) species formed at room temperature in situ from commercially available compounds (viz., InCl $\mathrm{I}_{3}, \mathrm{Sb}\left[\mathrm{NMe}_{2}\right]_{3}$ and a primary alkylamine) to obtain InSb adduct complexes. These complexes are successfully used as precursors for the synthesis of colloidal InSb QDs ranging from 2.8 to $18.2 \mathrm{~nm}$ in diameter by fast coreduction at sufficiently high temperatures $\left(\geq 230^{\circ} \mathrm{C}\right)$. Our findings allow us to propose a formation mechanism for the QDs synthesized in our work, which is based on a nonclassical nucleation event, followed by aggregative growth. This yields ensembles with multimodal size

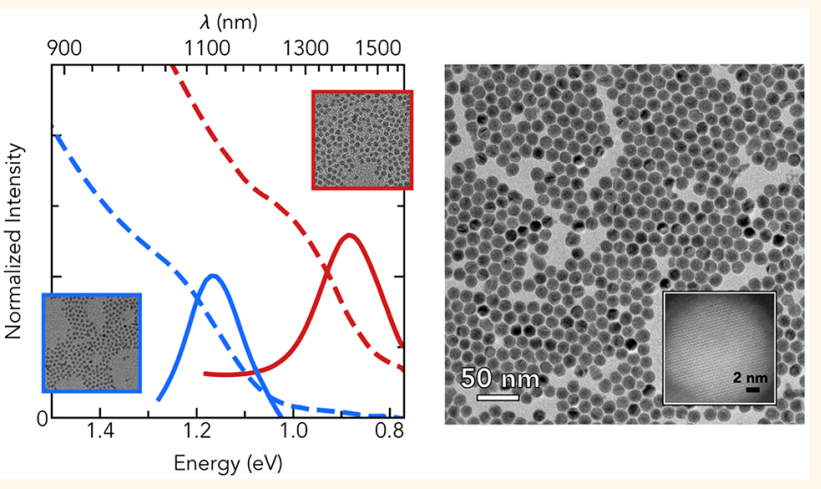
distributions, which can be fractionated in subensembles with relatively narrow polydispersity by postsynthetic size fractionation. InSb QDs with diameters below $7.0 \mathrm{~nm}$ have the zinc blende crystal structure, while ensembles of larger QDs $(\geq 10 \mathrm{~nm})$ consist of a mixture of wurtzite and zinc blende QDs. The QDs exhibit photoluminescence with small Stokes shifts and short radiative lifetimes, implying that the emission is due to band-edge recombination and that the direct nature of the bandgap of bulk InSb is preserved in InSb QDs. Finally, we constructed a sizing curve correlating the peak position of the lowest energy absorption transition with the QD diameters, which shows that the band gap of colloidal InSb QDs increases with size reduction following a $1 / d$ dependence.

KEYWORDS: indium antimonide, colloidal quantum dots, near-infrared emission, semiconductor nanocrystals, single-source precursor, III-V semiconductors

$\mathrm{T}$ he distinctive properties of indium antimonide $(\mathrm{InSb})$ make nanostructures of this III-V semiconductor promising material platforms for a large variety of optoelectronic devices that could potentially outperform those based on other semiconductors. InSb possesses a narrow direct band gap $(0.17 \mathrm{eV}$ at $300 \mathrm{~K})$ and the highest room temperature electron mobility $\left(7.7 \times 10^{4} \mathrm{~cm}^{2} / \mathrm{V} \cdot \mathrm{s}\right)$, the lowest thermal conductivity $\left(0.18 \mathrm{~W} / \mathrm{cm} \cdot{ }^{\circ} \mathrm{C}\right)$, and the smallest exciton binding energy $(0.5 \mathrm{meV})$ of all semiconductors, ${ }^{1}$ thus being ideal for infrared detectors, ${ }^{2}$ ultrafast electronics, ${ }^{3}$ and thermoelectric power conversion. ${ }^{4}$ Moreover, its giant gfactor $(-51.3)^{5}$ and strong spin-orbit coupling, in combination with its high electron mobility, are ideally suited for spintronic and quantum computing devices and have led in recent years to many breakthroughs, such as the demonstration of Majorana zero modes and spin-orbit qubits, which sparked a surge of interest in InSb nanowires. ${ }^{6-11}$
However, the potential of InSb nanostructures can only be fully exploited in colloidal quantum dots. Owing to the large exciton Bohr radius of $\mathrm{InSb}(61 \mathrm{~nm}),{ }^{1}$ quantum dots (QDs) of this material are obtained already at relatively large nanocrystal sizes and allow widely tunable bandgaps in the near-IR (NIR) spectral range through size control. Given the inherent advantages of colloidal synthesis methods, this would allow the use of colloidal InSb QDs as building blocks in low-cost, solution-processed optoelectronic devices, such as ultrafast

Received: June 7, 2020

Accepted: September 11, 2020

Published: September 11, 2020 
field-effect transistors, photodetectors, thermoelectric devices, LEDs, and solar cells. ${ }^{12}$ For example, colloidal InSb QDs are promising nontoxic alternatives for $\mathrm{Hg}$ - and $\mathrm{Pb}$-chalcogenide QDs in hyperspectral sensors in the short- to midwave infrared spectral window $(1.5-7.7 \mu \mathrm{m}){ }^{13}$ as labels for deep-tissue imaging in the second and third NIR biological windows (11.35 and $1.55-1.87 \mu \mathrm{m}$, respectively), ${ }^{14}$ in LEDs and lasers in telecommunications $\mathrm{O}$ - and E-bands $(\sim 1.3 \mu \mathrm{m})$, and in QDsolar cells. ${ }^{15}$ The latter application could also potentially benefit from carrier multiplication, which has been recently predicted to be efficient in InSb QDs. ${ }^{16}$ Moreover, InSb QDs have been theoretically predicted to show a strongly sizedependent electron g-factor, varying from -51.3 for bulk to zero for $4 \mathrm{~nm}$ diameter QDs, ${ }^{17}$ which further enhances their potential for quantum computing, since a null electron g-factor is interesting for coherent photon-to-spin conversion in qubits. $^{18}$

Despite the unparalleled potential of InSb nanostructures, their synthesis has proven to be very challenging, even in comparison with other III-V semiconductors, which are notoriously difficult to synthesize due to their strongly covalent nature, thereby requiring highly reactive precursors and high reaction temperatures. ${ }^{19}$ These requirements are particularly stringent for InSb, which is the most covalent of the III-V semiconductors. Furthermore, this compound is characterized by crystallographic facets with low surface free energy and slow growth rates. $^{20}$ Additional challenges when growing InSb nanostructures by vapor-phase techniques are posed by the low vapor pressure and surface energy of antimony and its surfactant properties. ${ }^{21}$ These challenges were nonetheless successfully addressed in the growth of InSb nanowires by metal organic vapor phase epitaxy (MOVPE), which has recently reached a very mature level, yielding arrays of highquality, monodisperse, stemless InSb nanowires (diameter: $70-170 \mathrm{~nm}$, length: $2-10 \mu \mathrm{m}) .^{21}$

In striking contrast, the synthesis of colloidal InSb QDs remains underdeveloped with respect to that of II-VI, IV-VI, and other III-V QDs, despite recent advances. ${ }^{22-24}$ It is widely accepted that this difficulty originates from the lack of suitable In and $\mathrm{Sb}$ precursors. Unfortunately, the colloidal synthesis of InSb QDs cannot benefit from the highly reactive precursors that were successfully used in the MOVPE synthesis of InSb nanowires (viz., trimethylindium and trimethylantimony) which were shown to lead to uncontrollable growth under the conditions prevalent in liquid-phase colloidal synthesis methods. ${ }^{23}$ We note that this is not surprising, since the success accomplished in the synthesis of InSb nanowires could only be achieved in a very narrow spot in the parameter space using conditions that are incompatible with colloidal synthesis methods (viz., gold-catalyzed vaporliquid-solid epitaxy, temperatures as high as $495{ }^{\circ} \mathrm{C}, \mathrm{Sb} / \mathrm{In}$ molar ratios ranging from 300 to 7000 , and low pressures). ${ }^{21}$ As a result, previous work on colloidal InSb QDs has been mostly focused on identifying precursors and conditions capable of yielding size and shape control similar to that currently available for the II-VI and IV-VI QDs.

However, most reaction schemes investigated to date failed to provide adequate size control. For example, in an attempt to extend dehalosilylation reactions to the InSb system, Evans et al. reacted tris(trimethylsilyl)antimony (a very reactive compound) with indium stearate (a relatively inert compound), but obtained only a polydisperse ensemble of $6 \pm 3$ $\mathrm{nm}$ diameter QDs. ${ }^{25}$ Later work by Reiss and co-workers investigated the hypothesis that the reaction kinetics would be better controlled if the precursors contained the elements already in their final oxidation states (i.e., $\mathrm{Sb}^{3-}$ and $\mathrm{In}^{3+}$ ) and therefore reacted stibine $\left(\mathrm{SbH}_{3}\right.$, a highly toxic and reactive gas) with indium myristate. ${ }^{26}$ However, size control remained elusive, as only InSb QDs of $8.8 \pm 0.8 \mathrm{~nm}$ diameter could be obtained, regardless of the conditions used. ${ }^{26}$ This reaction concept was recently revisited by Houtepen and co-workers, ${ }^{27}$ who opted for the generation of stibine in situ by reacting tris(dimethylamino)antimony with $n$-butyllithium, under the assumption that this would make the reaction kinetics more controllable due to the slower release of $\mathrm{SbH}_{3}$. The synthetic approach used by these authors thus consisted of the sequential injection of $\mathrm{Sb}\left[\mathrm{NMe}_{2}\right]_{3}$ and $n$-butyllithium into a solution of indium oleate. ${ }^{27}$ Nevertheless, only very small (1-2 $\mathrm{nm}$ diameter) and In-rich QDs were obtained, regardless of the reaction conditions. ${ }^{27}$

The first synthetic scheme to successfully achieve colloidal InSb QDs with size tunability $(3.4-6.5 \mathrm{~nm})$ and narrow polydispersity $(\sim 15 \%$, reduced to $\sim 5 \%$ by postsynthetic size fractionation) was developed by Talapin and co-workers. ${ }^{22}$ Their approach consisted of the coreduction of antimony silylamide $\left(\mathrm{Sb}\left[\mathrm{N}\left(\mathrm{Si}(\mathrm{Me})_{3}\right)_{2}\right]_{3}\right)$ and $\mathrm{InCl}_{3}$ to $\mathrm{Sb}^{0}$ and $\mathrm{In}^{0}$ by lithium triethylborohydride $\left(\mathrm{LiEt}_{3} \mathrm{BH}\right.$, also known as superhydride) at room temperature, followed by heating to the growth temperature $\left(240-260{ }^{\circ} \mathrm{C}\right){ }^{22}$ Subsequent work by Yarema and Kovalenko obtained InSb QDs with diameters in the $8-12 \mathrm{~nm}$ range ( $<10 \%$ polydispersity) by injecting a mixture of indium silylamide $\left(\operatorname{In}\left[\mathrm{N}\left(\mathrm{SiMe}_{3}\right)_{2}\right]_{3}\right)$ and tris(dimethylamino)antimony $\left(\mathrm{Sb}\left(\mathrm{NMe}_{2}\right)_{3}\right)$ into trioctylamine at $250{ }^{\circ} \mathrm{C} .^{23} \mathrm{~A}$ heating up variation of this reaction was also carried out, leading to sizes in the $4.6-7.2 \mathrm{~nm}$ range, but with larger size distributions $(\sim 15 \%) .{ }^{23}$ However, indium and antimony silylamides are not commercially available and must thus be directly synthesized and purified. Moreover, they are sensitive to oxygen, moisture, and light and therefore difficult to prepare and store. This affects the reproducibility of the synthesis and makes it more complicated and time-consuming, thereby increasing its cost.

In an attempt to synthesize colloidal InSb QDs using only commercially available compounds, Jeong and co-workers injected a mixture of $\mathrm{Li}\left[\mathrm{N}\left(\mathrm{SiMe}_{3}\right)_{2}\right]$ and $\mathrm{Sb}\left[\mathrm{NMe}_{2}\right]_{3}$ into a solution of $\mathrm{InCl}_{3}$ in trioctylamine at $250{ }^{\circ} \mathrm{C}$, but obtained only polydisperse $\mathrm{InSb} \mathrm{QDs}$ in the 3 to $6 \mathrm{~nm}$ diameter range, displaying featureless absorption spectra. ${ }^{28}$ This outcome can be attributed to an imbalance in the reduction kinetics of the two elements, with antimony reducing faster and forming $\mathrm{Sb}$ nanocrystals (NCs) at early stages of the reaction, which are later converted to polydisperse InSb NCs when the concentration of $\mathrm{In}^{0}$ becomes sufficiently high. ${ }^{28}$ Recently, a successful synthetic route to colloidal $\operatorname{In}\left(\mathrm{As}_{1-x} \mathrm{Sb}_{x}\right)(x=0-1)$ QDs based on the coreduction of commercially available Inand $\mathrm{Pn}$-precursors $(\mathrm{Pn}=\mathrm{As}, \mathrm{Sb})$ has been demonstrated by Kagan and co-workers. ${ }^{24}$ Their procedure involves mixing $\mathrm{InCl}_{3}$-oleylamine and $\mathrm{PnCl}_{3}$-oleylamine precursors (prepared by overnight heating at $50{ }^{\circ} \mathrm{C}$ ) at room temperature, followed by reduction with superhydride and heating up to the reaction temperature. ${ }^{24}$ The size of the InSb QDs could be tailored from 4.2 to $7.5 \mathrm{~nm}(\sim 10 \%$ polydispersity after postsynthetic size fractionation) by tuning the reaction temperature from 260 to $320{ }^{\circ} \mathrm{C}$. ${ }^{24}$

Here, we report the synthesis of colloidal InSb QDs ranging from 2.8 to $18.2 \mathrm{~nm}$ in diameter. The synthesized QDs in the 


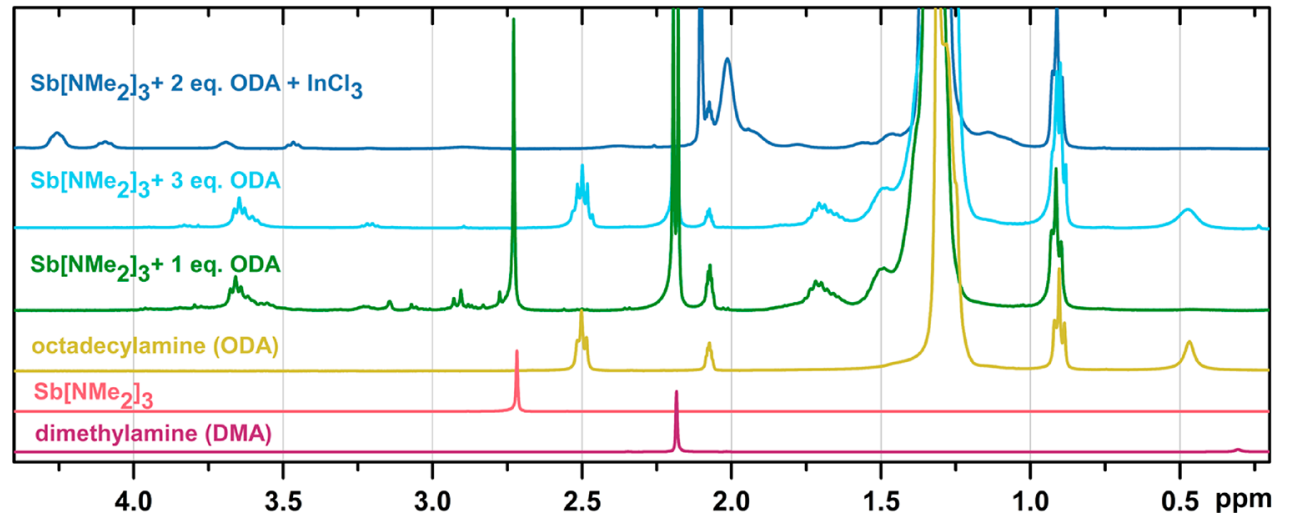

Figure 1. ${ }^{1} \mathrm{H}$ NMR spectra of the precursor solution, before and after the addition of $\mathrm{InCl}_{3}$. Spectra of the pure components are included for reference (DMA, $\mathrm{Sb}\left[\mathrm{NMe}_{2}\right]_{3}, \mathrm{ODA}$ ). The peak at $2.11 \mathrm{ppm}$ in the four top spectra originates from residual undeuterated toluene and is absent in the two bottom spectra because the concentration of these solutions was substantially higher. Additional ${ }^{1} \mathrm{H}$ and ${ }^{13} \mathrm{C}$ NMR spectra are provided in the Supporting Information (sections S1 and S2).

2.8-6.0 nm range exhibit photoluminescence (PL) at room temperature with fast radiative lifetimes (e.g., $71 \mathrm{~ns}$ for $3.7 \mathrm{~nm}$ diameter QDs with PL peak position at $1250 \mathrm{~nm}$ ). Our method is based on the fast coreduction of two commercially available compounds $\left(\mathrm{Sb}\left[\mathrm{NMe}_{2}\right]_{3}\right.$ and $\left.\mathrm{InCl}_{3}\right)$ by injection of a solution containing stoichiometric amounts of both compounds into $\mathrm{LiEt}_{3} \mathrm{BH}$ in oleylamine at $240{ }^{\circ} \mathrm{C}$. To circumvent the difficulties encountered in previous work by Jeong and coworkers using the same combination of chemicals as precursors (i.e., formation of $\mathrm{Sb} \mathrm{NCs}$ ), ${ }^{28}$ we exploited the Lewis acidbase nature of $\mathrm{Sb}\left[\mathrm{NMe}_{2}\right]_{3}$ and $\mathrm{InCl}_{3}$ to prepare in situ a "single-source" precursor containing $\mathrm{In}-\mathrm{Sb}$ bonds that are sufficiently strong to yield precursor solutions stable at room temperature. Our results show that the use of this $\mathrm{InSb}$ precursor solution kinetically favors the formation of InSb QDs over $\mathrm{Sb}$ and In NCs, provided the coreduction is sufficiently fast to outcompete the dissociation of the $\mathrm{In}-\mathrm{Sb}$ bond in the precursor complex (i.e., reaction temperatures must be $230{ }^{\circ} \mathrm{C}$ or higher). Moreover, we observe that the nucleation and growth of the InSb QDs proceed through nonclassical pathways (aggregative growth), yielding ensembles with multimodal size distributions, which can be fractionated in subensembles with relatively narrow $(\geq 10 \%)$ polydispersity by postsynthetic size-selective precipitation. The availability of QDs spanning a wide size range allowed us to construct a sizing curve, which shows that the band gap of colloidal $\mathrm{InSb}$ QDs increases with size reduction following a $1 / d$ dependence.

\section{RESULTS AND DISCUSSION}

InSb Precursor Solution. To synthesize colloidal InSb QDs, we make use of two commercially available compounds as precursors: $\mathrm{Sb}\left[\mathrm{NMe}_{2}\right]_{3}$ and $\mathrm{InCl}_{3}$ (see the Methods for details). Considering that the former is a Lewis base, while the latter is a Lewis acid, one would expect these compounds to form a Lewis acid-base adduct under suitable conditions. ${ }^{29}$ We observed that these conditions are, however, not met without adjuvant chemicals, since $\mathrm{InCl}_{3}$ does not dissolve in a solution of $\mathrm{Sb}\left[\mathrm{NMe}_{2}\right]_{3}$ in toluene. Nevertheless, if a specific amount (viz., $1.5-2.5$ equiv with respect to $\mathrm{Sb}\left[\mathrm{NMe}_{2}\right]_{3}$ ) of a primary amine (oleyl-, octadecyl-, hexadecyl-, dodecyl-, or octylamine) is added to the solution of $\mathrm{Sb}\left[\mathrm{NMe}_{2}\right]_{3}$ in toluene, its color changes from pale to bright yellow, indicating an interaction between the amine and the antimony compound. Interestingly, $\mathrm{InCl}_{3}$ is readily soluble at room temperature in the $\mathrm{Sb}\left[\mathrm{NMe}_{2}\right]_{3}$-amine solution, allowing us to prepare a precursor solution containing equimolar amounts of $\mathrm{InCl}_{3}$ and $\mathrm{Sb}\left[\mathrm{NMe}_{2}\right]_{3}$ (Supporting Information, Table S1), which is stable for at least several weeks under nitrogen atmosphere. Moreover, as recently demonstrated by Kagan and coworkers, ${ }^{24}$ the dissolution of $\mathrm{InCl}_{3}$ in primary amines requires overnight heating at $50{ }^{\circ} \mathrm{C}$ in neat amines and is thus negligible under the conditions used in our experiments (room temperature, diluted solutions, reaction time scale $<1 \mathrm{~min}$ ), unless both the primary amine and $\mathrm{Sb}\left[\mathrm{NMe}_{2}\right]_{3}$ are present in solution.

To understand the chemical nature of the InSb precursor solution, ${ }^{1} \mathrm{H}$ and ${ }^{13} \mathrm{C}$ NMR spectroscopy was used to investigate different $\mathrm{Sb}\left[\mathrm{NMe}_{2}\right]_{3}$-octadecylamine solutions, before and after the addition of $\mathrm{InCl}_{3}$. Although for convenience oleylamine (OLA) is used to prepare the $\mathrm{InSb}$ precursor in our optimized synthesis procedure (see the Methods for details), octadecylamine (ODA) was chosen for the NMR spectroscopy investigations because it gives the same outcome in the synthesis while being commercially available at higher purity ( $\geq 99.0 \%)$. Figure 1 shows the ${ }^{1} \mathrm{H}$ NMR spectra of the solutions and compounds of interest in deuterated toluene (toluene- $d_{8}$ ). Selected ${ }^{1} \mathrm{H},{ }^{13} \mathrm{C} \mathrm{NMR}$, and 2D NMR spectra, necessary to distinguish between $\mathrm{CH}_{2}$ and $\mathrm{CH}_{3}$ groups (Attached Proton Test, APT), to recognize couplings between different protons (homonuclear COrrelation spectroscopy, COSY), and to identify what protons belong to what carbon atoms (heteronuclear multiple-quantum correlation, HMQC) are included in the Supporting Information (sections $S 1$ and $\mathrm{S} 2$ and Figures S1_S8). In the spectrum of pure $\mathrm{Sb}\left[\mathrm{NMe}_{2}\right]_{3}$ (Figure 1) the only signal is a singlet at $2.75 \mathrm{ppm}$ from the protons of the methyl groups. The spectrum of pure dimethylamine (DMA, included because it is the protonated form of the organic groups bound to $\mathrm{Sb}$ in $\mathrm{Sb}\left[\mathrm{NMe}_{2}\right]_{3}$ ) presents a singlet at $2.18 \mathrm{ppm}$ and a broad signal at $0.3 \mathrm{ppm}$ from the $-\mathrm{NH}$ group (Figure 1). The dimethylamino signal in $\mathrm{Sb}\left[\mathrm{NMe}_{2}\right]_{3}$ is shifted downfield with respect to free DMA, due to the electron-withdrawing effect of $\mathrm{Sb}$. The spectrum of pure ODA (Figure 1) is characterized by a triplet at $2.53 \mathrm{ppm}$ from the $-\mathrm{CH}_{2}$ group directly bound to the nitrogen atom. Additionally, a very intense multiplet centered at $1.3 \mathrm{ppm}$ from the internal $-\mathrm{CH}_{2}$ groups of the octadecyl chain, a triplet at $0.95 \mathrm{ppm}$ from the terminal $-\mathrm{CH}_{3}$ group and a broad signal 
at $0.45 \mathrm{ppm}$ from the $-\mathrm{NH}_{2}$ group are also present (the signal at $2.11 \mathrm{ppm}$ is from residual nondeuterated toluene).

After the addition of one equivalent of ODA to $\mathrm{Sb}\left[\mathrm{NMe}_{2}\right]_{3}$ in toluene- $d_{8}$ (Figure 1), the signal from free DMA appears at 2.21-2.23 ppm, while the signal assigned to $\mathrm{N}-\mathrm{CH}_{2}$ in free ODA (2.53 ppm) is not observed, and that of $\mathrm{Sb}\left[\mathrm{NMe}_{2}\right]_{3}$ $(2.76 \mathrm{ppm})$ is still present. Further, additional signals become visible between 3.2 and $3.9 \mathrm{ppm}$. We note that the signal from free DMA appears as a doublet in the ${ }^{1} \mathrm{H}$ NMR spectrum of the $\mathrm{Sb}\left[\mathrm{NMe}_{2}\right]_{3}$-ODA solutions due to the ${ }^{3} J$ coupling between the protons of the methyl group and the proton bound to $\mathrm{N}$. This coupling emerges only under anhydrous conditions and is therefore absent in the reference sample (not anhydrous), resulting in a singlet. When 1.65 equiv of ODA is added to $\mathrm{Sb}\left[\mathrm{NMe}_{2}\right]_{3}$ in toluene- $d_{8}$ (Figure $\mathrm{S} 1$ ), the signal from $\mathrm{Sb}\left[\mathrm{NMe}_{2}\right]_{3}(2.76 \mathrm{ppm})$ is no longer present, while a very weak signal from free ODA $(2.53 \mathrm{ppm})$ is observed and the signal from free DMA and the peaks between 3.2 and $3.9 \mathrm{ppm}$ remain unaffected. The spectrum remains essentially unchanged after the addition of 2 and 3 equiv of ODA to $\mathrm{Sb}\left[\mathrm{NMe}_{2}\right]_{3}$ in toluene- $d_{8}$ (Figure 1 ), except for the signal from free ODA (2.53 ppm), which becomes more intense. Considering that equilibria of the following type are expected

$$
\begin{aligned}
& x \mathrm{Sb}\left[\mathrm{NMe}_{2}\right]_{3}+(y+z) \mathrm{H}_{2} \mathrm{NR} \\
& \quad \leftrightarrow \mathrm{Sb}_{x}\left[\mathrm{NMe}_{2}\right]_{(3 x-y-z)}[\mathrm{HNR}]_{y}[\mathrm{NR}]_{z}+(y+z) \mathrm{HNMe}_{2}
\end{aligned}
$$

the peaks appearing between 3.2 and $3.9 \mathrm{ppm}$ are assigned to protons from the $\mathrm{N}-\mathrm{CH}_{2}$ groups of ODA bound to $\mathrm{Sb}$. COSY NMR (Figure S2) corroborates this assignment. As expected, these protons are less shielded after the binding of the amine to $\mathrm{Sb}$, which withdraws electron density, similar to what is observed for the methyl groups of DMA (Figure 1).

The presence of these new peaks in different positions suggests that either more than one species form or one species forms in which ODA is present in different configurations, for example, as both terminal and bridging ligand. The appearance of the peak of free DMA indicates that at least one dimethylamino group has been replaced by ODA. However, the absence of the signal for bound DMA implies that all three DMA groups of the original $\mathrm{Sb}\left[\mathrm{NMe}_{2}\right]_{3}$ have been replaced by ODA. The unchanged position and intensity of the peaks between 3.2 and $3.9 \mathrm{ppm}$ (assigned to protons from the $\mathrm{N}$ $\mathrm{CH}_{2}$ groups of ODA bound to $\mathrm{Sb}$ ) with increasing amounts of amine, along with the appearance of the free ODA peak after addition of more than 1.5 equiv of ODA, suggest that the amine-antimony complex in solution has no more than 3 ODA ligands for every $2 \mathrm{Sb}$ atoms, even at higher amine concentrations. This is consistent with the ratio between "bound" and "free" ODA observed in the sample with 3 equiv of ODA (viz., 0.9, which can be calculated from the ratio between the integrals of the peaks assigned to the $\mathrm{N}-\mathrm{CH}_{2}$ groups for "bound" and "free" ODA, Figure S3). Taken together, these observations suggest that ODA may be present only as an amido bridging ligand, which is consistent with the lack of signals originating from $\mathrm{N}-\mathrm{H}$ protons from "bound" ODA in the COSY spectrum (Figure S2). Several compounds of $\mathrm{Sb}(\mathrm{III})$ with primary amines are known, ${ }^{30-32}$ which are usually dimers or heterocycles. In these complexes, the protons of methylene groups bound to $\mathrm{N}$ are also observed to shift downfield, ${ }^{31}$ while the signal from bound DMA (if still present) is found at the same value of chemical shift of $\mathrm{Sb}\left[\mathrm{NMe}_{2}\right]_{3}{ }^{30}$ Based on the examples from the literature, and the information obtained from the NMR spectra discussed above, we propose that species such as $\mathrm{Sb}_{x}[\mathrm{ODA}]_{y}(x=2$ or 4 , $y=3$ or 6 , respectively) likely coexist in solution (see Supporting Information, Scheme S1). It is interesting to note that imidoantimony macrocyclic oligomers with these stoichiometries are known and have been well-characterized by both NMR spectroscopy and X-ray crystallography. ${ }^{33,34}$ Additionally, As-based compounds with primary linear amines $\left(\mathrm{As}_{4}\left(\mathrm{NCH}_{3}\right)_{6}\right)$ have been reported, adopting a structure which corresponds to that proposed in Scheme S1-B. ${ }^{35}$ It is worth noting that the transamination between the secondary amino groups of the pnictide precursor and the solvent (a primary amine) has been recognized to be the first step in the formation mechanism of $\mathrm{InP}$ quantum dots, starting from $\mathrm{P}\left[\mathrm{NMe}_{2}\right]_{3}$ and $\mathrm{InCl}_{3}$ as precursors. ${ }^{36}$

As mentioned above, when an equimolar amount of $\mathrm{InCl}_{3}$ is added to the $\mathrm{Sb}\left[\mathrm{NMe}_{2}\right]_{3}$-amine solution, a clear pale yellow solution is formed that is stable for at least several weeks. The ${ }^{1} \mathrm{H}$ NMR spectrum of a solution containing $\mathrm{Sb}\left[\mathrm{NMe}_{2}\right]_{3}$ with 2 equiv of ODA and 1 equiv of $\mathrm{InCl}_{3}$ is presented in Figure 1, showing that after addition of $\mathrm{InCl}_{3}$ all peaks assigned to $\mathrm{N}$ $\mathrm{CH}_{2}$ of ODA bound to $\mathrm{Sb}$ move to higher ppm values, appearing as four triplets between 3.4 and $4.3 \mathrm{ppm}$. Interestingly, free DMA is not observed, while a signal from "bound" DMA, which is absent in solutions with only $\mathrm{Sb}\left[\mathrm{NMe}_{2}\right]_{3}$ and ODA, appears as a broadened peak at $\sim 2$ ppm (a chemical shift that is lower than those of both free DMA and bound DMA in $\mathrm{Sb}\left[\mathrm{NMe}_{2}\right]_{3}$, i.e., $2.21-2.23$ and 2.76 ppm, respectively). The NMR spectra that support these assignments are shown in Figures S4-S8. As a control experiment to verify the role of DMA, a solution of $\mathrm{Sb}\left[\mathrm{NMe}_{2}\right]_{3}$ and ODA was completely dried under vacuum and subsequently recomposed by adding the same volume of toluene to the dry residue. This recomposed solution was found to be unable to dissolve $\mathrm{InCl}_{3}$, in contrast with the original solution, despite the fact that both solutions contain the same concentration of ODA and $\mathrm{Sb}$. Considering that both toluene and free DMA evaporate under vacuum, but only toluene was added to the dried residue, we conclude that free DMA is also necessary to solubilize $\mathrm{InCl}_{3}$, possibly by (partially) exchanging the chlorides bound to the Indium atom. However, this cannot account for the downfield shift of the signal of the $\mathrm{N}-\mathrm{CH}_{2}$ protons of ODA bound to $\mathrm{Sb}$ after addition of $\mathrm{InCl}_{3}$ (from 3.2-3.9 to 3.4 and $4.3 \mathrm{ppm}$ ), since the solubilization of $\mathrm{InCl}_{3}$ by formation of complexes containing only DMA would leave this signal unaffected, while a direct interaction between $\mathrm{InCl}_{3}$ and ODA would lead to an upfield shift and/or the appearance of a new signal due to the $\mathrm{N}-\mathrm{CH}_{2}$ protons of ODA bound to In. For comparison, we note that the $\mathrm{N}-\mathrm{CH}_{2}$ signal of OLA (at $2.68 \mathrm{ppm}$ for the free amine) has been reported to shift to $3.26 \mathrm{ppm}$ for OLA bound to $\mathrm{SbCl}_{3}$ and to 2.79 for OLA bound to $\mathrm{InCl}_{3}{ }^{24}$

On the other hand, the downfield shift of the signal of ODA bound to $\mathrm{Sb}$ supports the notion that a Lewis acid-base interaction between the $\mathrm{Sb}$ (III) and the $\mathrm{In}(\mathrm{III})$ complexes present in solution (viz., $\mathrm{Sb}_{x}[\mathrm{ODA}]_{y}$ with $x=2$ or $4, y=3$ or 6 species as Lewis bases and $\mathrm{InCl}_{3-z} \mathrm{DMA}_{z}$ as Lewis acids) has led to the formation of adducts containing a direct $\mathrm{Sb}$-In bond, since the formation of such a bond would reduce the shielding of all $\mathrm{Sb}-\mathrm{N}-\mathrm{CH}_{2}$ protons due to shifting of electron density toward the In atom. The literature contains several examples of compounds formed through Lewis acid-base interactions between Sb-based Lewis bases and In-based Lewis 
acids that contain $\mathrm{In}-\mathrm{Sb}$ bonds [e.g., $\left(\mathrm{Me}_{3} \mathrm{SiCH}_{2}\right)_{3} \mathrm{In} \cdot \mathrm{Sb}$ $\left.\left(\mathrm{SiMe}_{3}\right)_{3}\right]^{29,37,38}$ These compounds have been extensively characterized by solution ${ }^{1} \mathrm{H}$ and ${ }^{13} \mathrm{C}$ NMR and, in a few cases, also X-ray crystallography, which revealed $\mathrm{In}-\mathrm{Sb}$ bond lengths in the 288-300.8 pm range. ${ }^{29,37,38}$ Dimers and 6-membered heterocycles are common, and often the reactions yield a mixture of compositionally and structurally distinct species. ${ }^{29}$ The formation of these compounds is driven by electronic effects, but is strongly constrained by the steric demands of the species involved. ${ }^{29}$ We thus propose that in the present case the Lewis acid-base interaction between $\mathrm{InCl}_{3}$ and $\mathrm{Sb}$ $\left[\mathrm{NMe}_{2}\right]_{3}$ is initially severely hindered by steric repulsion between the ligands due to the fact that the secondary amino groups on the $\mathrm{Sb}$ atom are bulky. The replacement of these groups by primary amino groups upon formation of the $\mathrm{Sb}_{x}[\mathrm{ODA}]_{y}(x=2$ or $4, y=3$ or 6$)$ and $\mathrm{InCl}_{3-z} \mathrm{DMA}_{z}$ species allows for a more favorable Lewis acid-base interaction, since primary amino groups are less bulky than secondary ones, which facilitates access to the $\mathrm{Sb}$ atom. Most likely, the reaction does not yield a single product but rather a mixture of related compounds with different compositions and/or structures, albeit with a $1: 1 \mathrm{In} / \mathrm{Sb}$ ratio in all cases. To determine the exact composition and structure of these $\mathrm{In}-\mathrm{Sb}$ heteroamino and amido complexes, and the nature and strength of the In-Sb bonds, further experiments are required using sophisticated structural characterization techniques, such as X-ray crystallography and X-ray absorption spectroscopy (in particular extended X-ray absorption fine structure, EXAFS, and X-ray absorption near edge structure, XANES), which are beyond the scope of the current work. Nevertheless, the results presented above support the formation of a family of aminoand amido-complexes containing $\mathrm{In}-\mathrm{Sb}$ bonds that are stable in solution at room temperature, which makes them viable "single-source" precursors for the synthesis of stoichiometric InSb QDs. For simplicity, we will hereafter refer to the solution of these compounds as the "InSb precursor solution".

Synthesis of Colloidal InSb QDs. Our optimized protocol to synthesize colloidal InSb QDs consists of injecting $0.25 \mathrm{~mL}$ of the $\mathrm{InSb}$ precursor solution $([\mathrm{InSb}]=0.3 \mathrm{M})$ into $10 \mathrm{~mL}$ of OLA at $240{ }^{\circ} \mathrm{C}, 2$ to $10 \mathrm{~min}$ after $\mathrm{LiEt}_{3} \mathrm{BH}$ (superhydride, $\mathrm{SH}$ ) has been added in molar ratios ranging from 9:1 to $15: 1$ (stoichiometric $\mathrm{SH}: \mathrm{InSb}$ ratio is 6:1) (see the Methods for details). The outcome of the synthesis is the same regardless of the primary amine used to prepare the precursor solution and as solvent, provided its boiling point is sufficiently high. Nevertheless, OLA is preferred for convenience since it is liquid at room temperature despite its high boiling point. The relevance of the different time intervals after the addition of $\mathrm{SH}$ and of the different $\mathrm{SH} / \mathrm{InSb}$ ratios will be discussed in more detail below. After the precursor injection, the solution turns brown and then black within $10 \mathrm{~s}$, indicating that the formation of $\mathrm{InSb} \mathrm{QDs}$ is a very fast process. The reaction is allowed to proceed for a few $(1-5)$ minutes, after which it is quenched by dilution into cold toluene (2:1 volume ratio). To neutralize unreacted $\mathrm{SH}$ and to improve the colloidal stability of the synthesized InSb QDs, oleic acid is added to the crude reaction solution after its quenching in toluene. The reaction product is washed by precipitation with isometric butanol-methanol followed by centrifugation and resuspension in toluene (see the Methods for details). This procedure yields stoichiometric InSb QDs with average diameters ranging from 2.8 to $18.2 \mathrm{~nm}$ (polydispersity ranging from $20 \%$ to $6 \%$ ). The size polydispersity of the samples can be reduced to $\leq 10 \%$ by postsynthetic size-selective precipitation (Figure 2 and Figure S9, see the Methods for details). In the following, we will first

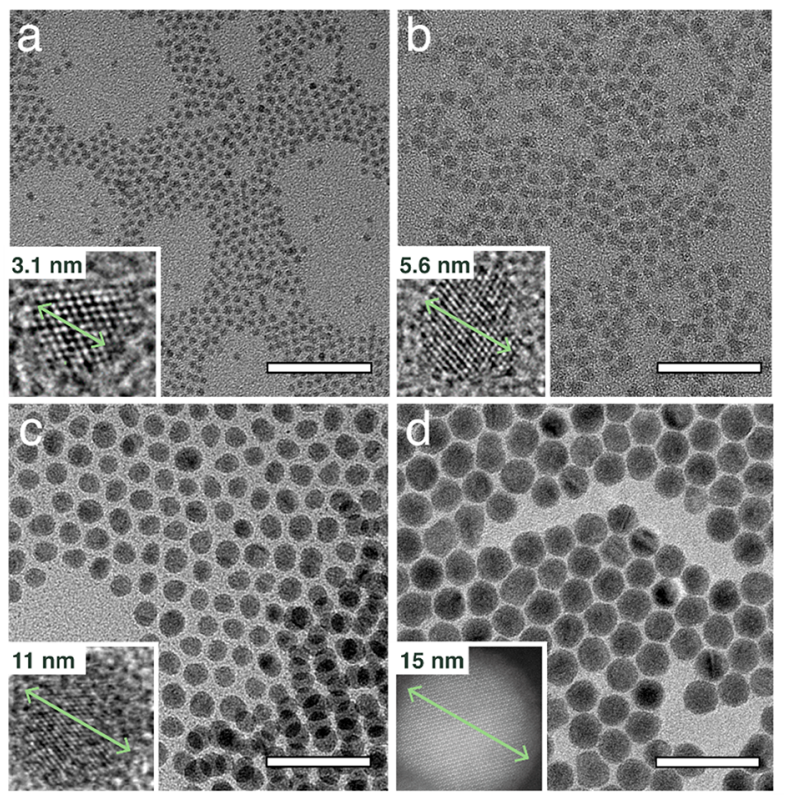

Figure 2. Overview TEM images of representative InSb QD samples after postsynthetic size fractionation: (a) $2.8 \pm 0.3 \mathrm{~nm}$, (b) $5.3 \pm 0.5 \mathrm{~nm},(\mathrm{c}) 10.3 \pm 1.2 \mathrm{~nm}$, and (d) $14.9 \pm 1.0 \mathrm{~nm}$. Highresolution TEM $(a-c)$ and STEM $(d)$ images of one exemplary QD for each ensemble are shown in the insets. The scalebars correspond to $50 \mathrm{~nm}$. Overview TEM images of $18.2 \pm 1.6 \mathrm{~nm}$ diameter InSb QDs are provided in the Supporting Information (Figure S9).

discuss the InSb QD samples obtained directly after the synthesis, without any size fractionation, and later address in more detail the properties of the QD samples obtained after the size-selective precipitation.

In the absence of superhydride, under otherwise identical conditions, the precursor injection results in the formation of large $(100-300 \mathrm{~nm}) \mathrm{Sb}$ NCs (Figure S10), showing that antimony is more readily reduced than indium, so that even a mild reducing agent such as oleylamine can reduce it at sufficiently high temperatures. This observation is consistent with previous work by $\mathrm{He}$ et al. on the preparation of colloidal metallic nanoparticles, which demonstrated that the formation of In nanoparticles from $\mathrm{InCl}_{3}$ in OLA requires superhydride, while $\mathrm{Sb}$ nanoparticles can be formed with milder reducing agents. ${ }^{39}$ This highlights that balancing the reduction rates of the two elements is of paramount importance to ensure that coreduction of In and Sb occurs. The use of a strong reducing agent such as superhydride is thus a necessary condition to obtain InSb QDs, but its effectiveness is crucially dependent on the reaction temperature. We observed that stoichiometric InSb QDs are only obtained at sufficiently high temperatures (above $230{ }^{\circ} \mathrm{C}$ ). At $210^{\circ} \mathrm{C}$, the synthesis product consists of a mixture of nearly spherical In-rich ( $80 \%$ In, $20 \% \mathrm{Sb})$ NCs and irregularly shaped Sb-rich (15\% In, $85 \% \mathrm{Sb}$ ) NCs (Figure $\mathrm{S} 11)$. At lower temperatures the imbalance in the composition of the NCs becomes even more pronounced.

These observations suggest that $[\mathrm{InSb}]_{n}$ units can only be formed from the InSb precursor if the reduction rates of both $\mathrm{Sb}$ and In are sufficiently fast to outcompete the dissociation of the "InSb" bonds in the precursor complex(es). If the 

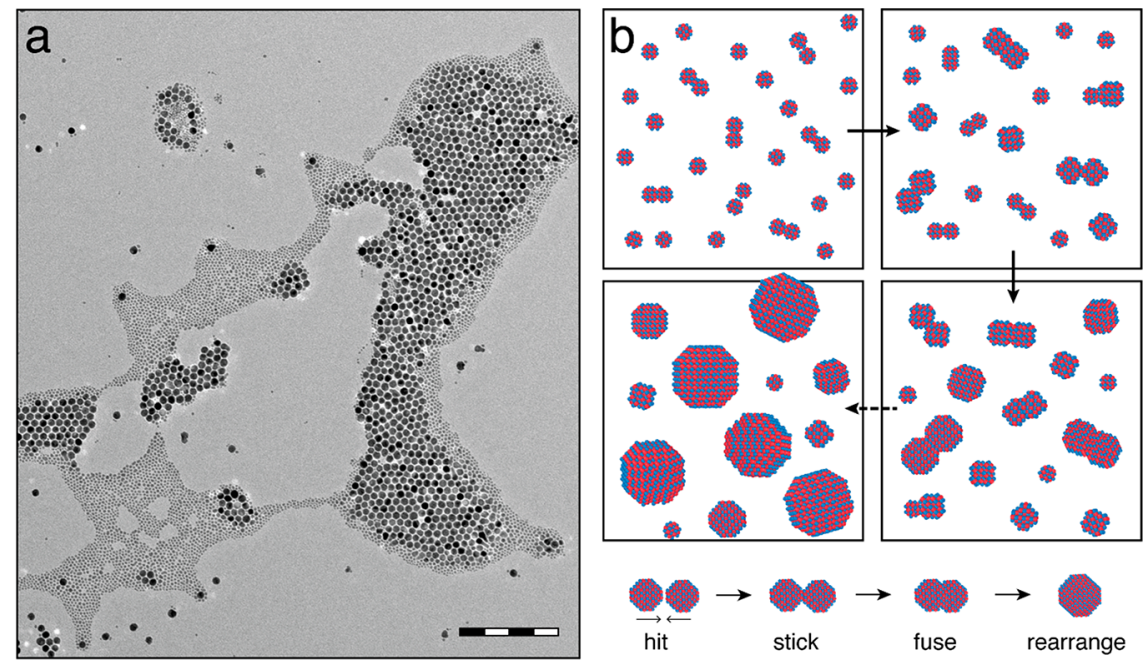

Figure 3. (a) Representative TEM image of a colloidal InSb QD sample prepared using a delay time of 10 min between the addition of superhydride to OLA at $240{ }^{\circ} \mathrm{C}$ and the injection of the InSb precursor solution. (b) Schematic illustration of the aggregative growth mechanism.

reduction rate of $\mathrm{Sb}$ is significantly faster than that of In, the InSb precursor will immediately dissociate since the interaction between $\mathrm{Sb}^{0}$ and an In-based Lewis acid is negligibly weak. This kinetic imbalance in the reduction rates leads to the independent formation of $\mathrm{Sb}^{0}$ and $\mathrm{In}^{0}$ species. As previously proposed by both Talapin and co-workers ${ }^{22}$ and Kagan and coworkers, ${ }^{24}$ these species are very reactive at high temperatures and act as monomers for the nucleation and growth of $\mathrm{InSb}$ QDs. However, $\mathrm{Sb}^{0}$ and $\mathrm{In}^{0}$ can also lead to nucleation and growth of metallic $\mathrm{Sb}$ and In NCs, which will compete with the formation of InSb QDs. Therefore, if Sb reduces faster than In, $\mathrm{Sb}$ NCs will unavoidably form at early stages of the reaction, as indeed observed by Tamang et al. ${ }^{28}$ after coinjection of $\mathrm{Sb}\left[\mathrm{NMe}_{2}\right]_{3}$ and $\mathrm{Li}\left[\mathrm{N}\left(\mathrm{SiMe}_{3}\right)_{2}\right]$ into a solution of $\mathrm{InCl}_{3}$ in trioctylamine at $250{ }^{\circ} \mathrm{C}$. Antimony NCs can be converted at later stages of the reaction to (Sb-rich) InSb NCs by reaction with either $\operatorname{In}^{0}$ species or liquid droplets of metallic In (melting point of indium: $156.6{ }^{\circ} \mathrm{C}$ ). Liquid droplets of indium may also dissolve smaller Sb NCs, forming In-rich NCs upon cooling, consistent with our observations for reactions at temperatures below $230{ }^{\circ} \mathrm{C}$ (Figure S11). The imbalance between the reduction kinetics of In and $\mathrm{Sb}$ is less apparent when the coreduction is carried out with superhydride at room temperature (as reported in refs 22 and 24) because at low temperatures $\mathrm{Sb}^{3+}$ is reduced to stable polyatomic structures such as $\mathrm{Sb}_{7}{ }^{3-24}$, which will not lead to the formation of metallic $\mathrm{Sb}$ NCs, allowing for a more controllable reaction between reduced $\mathrm{In}$ and $\mathrm{Sb}$ species upon heating up to the reaction temperature. In an attempt to compensate for the faster reduction kinetics of $\mathrm{Sb}$, Tamang et $\mathrm{al}^{28}$ first injected superhydride into a solution of $\mathrm{InCl}_{3}$ in trioctylamine at 250 ${ }^{\circ} \mathrm{C}$ and only $1 \mathrm{~min}$ later injected a substoichiometric amount of $\mathrm{Sb}\left[\mathrm{NMe}_{2}\right]_{3}(\mathrm{Sb} / \mathrm{In}=0.5)$. This resulted in large $(21 \mathrm{~nm})$ and polydisperse InSb NCs, which were nonetheless accompanied by $\mathrm{Sb} \mathrm{NCs}{ }^{28}$

We propose that, if the reduction rates of $\mathrm{In}$ and $\mathrm{Sb}$ are sufficiently fast to generate their zerovalent states while the InSb bonds in the precursor complex(es) are still intact, an $\mathrm{In}-\mathrm{Sb}$ covalent bond will immediately form through transfer of electron density from $\operatorname{In}^{0}$ to $\mathrm{Sb}^{0}$, so that they reach their final formal oxidation states of +3 and -3 , respectively. In this way, the InSb precursor complexes would be directly converted to $[\mathrm{InSb}]_{n}$ clusters, which can act as both nuclei and monomers for growth of InSb QDs. This would make the formation of InSb QDs kinetically favored over the competing formation of metallic In and Sb NCs. Evidence for the formation of $[\mathrm{InSb}]_{n}$ clusters at early stages of the reaction is provided by the absorption spectra of aliquots collected $\sim 2 \mathrm{~s}$ after the InSb precursor injection, which exhibit a broad peak at $\sim 370 \mathrm{~nm}$ $(3.4 \mathrm{eV})$ (Figure S12). Using a sizing curve developed in our work (see Optical Properties of Colloidal InSb QDs for further details), we reason that the absorption spectra of the early stage samples may be assigned to $[\mathrm{InSb}]_{n}$ molecular clusters with $n \sim 2.6$. This estimated $n$ is consistent with the proposed stoichiometry of the single-source $[\mathrm{InSb}]_{x}$ precursor complexes $(x=2$ and/or 4 , see InSb Precursor Solution above for a detailed discussion) and thus supports our hypothesis that at sufficiently high temperatures $\left(\geq 230^{\circ} \mathrm{C}\right)$, and in the presence of strong reducing agents (superhydride), the reduction kinetics of both In and $\mathrm{Sb}$ are sufficiently fast to allow coreduction of both elements while the InSb bonds in the precursor complex(es) are still intact, thereby converting them directly to $[\mathrm{InSb}]_{n}$ molecular clusters. We note that singlesource InSb precursors, such as $\left[t-\mathrm{Bu}_{3} \mathrm{In} * \mathrm{Sb}\left(\mathrm{SiMe}_{3}\right)_{3}\right]$, have been used in the past to synthesize nanocrystalline InSb by thermolysis at high temperatures $\left(350{ }^{\circ} \mathrm{C}\right)$, albeit with poor control over the size and aggregation of the nanocrystals, most likely because the reactions were carried out under vacuum. $^{29,38}$

Interestingly, despite its decisive role in the outcome of the synthesis, the reaction temperature was found to be of limited use in tailoring the size and polydispersity of the product $\mathrm{InSb}$ QDs, at least over the relatively narrow temperature range investigated in our work $\left(230-260{ }^{\circ} \mathrm{C}\right)$. Additionally, the QD sizes do not markedly change with increasing reaction time from 1 to $5 \mathrm{~min}$, implying that the InSb QD growth is a very fast process. The relatively minor impact of the reaction temperature and time on the final size of the colloidal $\mathrm{InSb}$ QDs synthesized in our work is in striking contrast with the behavior observed for colloidal QDs of other compositions (most notably II-VI and IV-VI semiconductors), ${ }^{40}$ but is in line with several previous reports on the synthesis of colloidal 
InSb QDs. ${ }^{25-28}$ On the other hand, the molar ratio between the amount of superhydride ( $\mathrm{SH}$ ) and InSb precursor injected in the reaction medium provides a very effective way to tailor the size of the product InSb QDs, with larger SH:InSb ratios resulting in smaller QDs (Figure S13). Talapin and co-workers also reported a correlation between the $\mathrm{SH}: \mathrm{InSb}$ ratio and the size of the InSb QDs, but observed the opposite trend (viz., larger InSb QDs for larger $\mathrm{SH}: \mathrm{InSb}$ ratios). ${ }^{22}$ A possible explanation for this difference is that the coreduction of In and $\mathrm{Sb}$ by $\mathrm{SH}$ addition was carried out at drastically different temperatures in the two works (room temperature in ref 22 and $240{ }^{\circ} \mathrm{C}$ in our work), which probably results in different formation mechanisms, as will be discussed in more detail below.

Another effective way to control the size of the InSb QDs obtained by the synthesis protocol developed in our work is to tune the time delay between the addition of SH to OLA at 240 ${ }^{\circ} \mathrm{C}$ and the injection of the $\mathrm{InSb}$ precursor solution. If this delay is increased from 2 to $10 \mathrm{~min}$, the mean size and size distribution of the product QDs changes dramatically, with shorter delays yielding ensembles of smaller InSb QDs with larger polydispersity and accompanied by a small fraction of larger nonstoichiometric nanoparticles (Figure S14), while longer delays yield InSb QDs displaying a markedly bimodal size distribution, without nonstoichiometric byproduct nanoparticles. A representative example of the latter case is provided in Figure 3, which shows that a sample obtained after a $10 \mathrm{~min}$ delay consists of two distinct subensembles of InSb QDs, one with smaller sizes and larger polydispersity $(5.6 \pm 0.8 \mathrm{~nm}$, i.e., $15 \%$ polydispersity) and one with significantly larger sizes but smaller polydispersity $(14.9 \pm 1.0 \mathrm{~nm}$, i.e., $7 \%$ polydispersity $)$. This striking observation provides valuable insights in the formation mechanism of the InSb QDs synthesized in our work, as will be clear in the discussion below.

Formation Mechanism of Colloidal InSb QDs. The results discussed above show that by tuning the $\mathrm{SH} / \mathrm{InSb}$ ratio $(9: 1-15: 1)$ and the time delay between the $\mathrm{SH}$ addition and the InSb precursor injection $(2-10 \mathrm{~min})$ we can synthesize ensembles of colloidal InSb QDs with mean diameters ranging from 2.8 to $18.2 \mathrm{~nm}$, without changing the reaction temperature $\left(240{ }^{\circ} \mathrm{C}\right)$ and time $(\sim 2 \mathrm{~min})$, provided the reaction temperature is sufficiently high $\left(\geq 230{ }^{\circ} \mathrm{C}\right)$. Lower reaction temperatures lead to mixtures of highly polydisperse In-rich and Sb-rich NCs, while temperatures in the 230-260 ${ }^{\circ} \mathrm{C}$ range successfully yield InSb QDs but have a minor impact on the final QD size. We observe that larger $\mathrm{SH} / \mathrm{InSb}$ ratios yield smaller mean diameters, while increasing the time delay between the $\mathrm{SH}$ addition and the InSb precursor injection has a striking impact on both the mean size, which becomes larger, and the size polydispersity, which evolves to a bimodal distribution consisting of distinct $\mathrm{QD}$ subensembles. Taken together, these observations allow us to propose a formation mechanism for the colloidal InSb QDs synthesized in our work. This mechanism is fundamentally different from those commonly observed for colloidal QDs of other semiconductor families (II-VI, IV-VI, III-V, and I-III-VI $)^{40-44}$ but bears strong similarities with that of colloidal NCs of metals under strongly reducing conditions. ${ }^{45-49}$ Although further studies will be needed to fully elucidate the mechanistic details, the currently available data are enough to support the key aspects of the proposed mechanism.

The first elementary kinetic step in the synthesis of colloidal $\mathrm{NCs}$ is the precursor to monomer conversion, which is followed by nucleation when the monomer concentration becomes sufficiently high. ${ }^{40}$ This is often the rate-limiting step in the synthesis of semiconductor NCs and thereby coexists with the subsequent nucleation and growth steps. ${ }^{40-43,50}$ The formation of $\mathrm{InSb}$ QDs dramatically deviates from this behavior, since the precursors are fully converted to monomers by a very fast coreduction reaction, which completely depletes the precursor reservoir. We argued above that at sufficiently high temperatures $\left(\geq 230{ }^{\circ} \mathrm{C}\right)$ the coreduction kinetics are sufficiently fast to directly convert the InSb precursor complex(es) to $[\mathrm{InSb}]_{n}$ molecular clusters. Evidence for the presence of such clusters at early stages of the reaction was discussed above (Figure S12). Given the highly covalent nature of $\mathrm{InSb}$ and its insolubility in the reaction medium, we propose that the formation of the $[\mathrm{InSb}]_{n}$ clusters can be seen as a nonclassical nucleation event, ${ }^{51}$ which immediately triggers further nanocrystal growth.

The observation of bimodal size distributions (Figure 3a) clearly demonstrates that the growth of the InSb QDs proceeds by aggregation and coalescence (Figure $3 \mathrm{~b}$ ). Bimodal size distributions are theoretically predicted for aggregative growth processes $^{51-54}$ and have been reported by many groups, notably during the synthesis of metal NCs ( $\mathrm{Pt}, \mathrm{Au}, \mathrm{Bi}, \mathrm{Pd}, \mathrm{Ag}$, $\left.\mathrm{Pt}_{3} \mathrm{Fe}\right){ }^{45-49,52}$ and also directly observed in real time by in situ TEM studies. ${ }^{48,49}$ Further support for this growth mechanism is given by the observation of a small fraction of InSb NCs that clearly consist of two attached InSb QDs (Figures S15 and S16, the different crystal structures will be discussed in more detail in the next section). We propose that these dimer QDs are intermediates in the aggregative growth process, being formed immediately after two InSb QDs with the same crystallographic orientation have coalesced, prior to their structural reshaping and refaceting into a more isotropic QD. An additional piece of evidence supporting the hypothesis of an aggregative growth mechanism comes from the occasional observation of QDs displaying twin boundary defects (Figure S17), which likely originate from the coalescence of two misoriented InSb QDs. Similar nanostructures (both dimers and NCs with a twin boundary) have been observed by in situ HR-TEM studies of the aggregative growth of Pt NCs using graphene liquid cells. ${ }^{48,49}$ Aggregative growth is the dominant growth mechanism when the monomer-supplying reaction is much faster than the overall growth rates, ${ }^{52}$ which is in line with the very fast InSb precursor to monomer conversion rates. This is also consistent with the fact that the InSb clusters and NCs present in solution are expected to interact strongly with one another, forming strong covalent bonds upon surface contact, while interacting only weakly with the ligands present in the reaction medium.

Aggregative growth starts with the fast formation of an ensemble of small, primary NCs which are colloidally unstable due to their large surface/volume ratio and high surface free energies. ${ }^{52}$ Owing to their high mobilities these NCs undergo multiple collisions with their neighbors, aggregating and coalescing with them when the collisions result in direct NC contact. The coalescence event results in a larger, more stable $\mathrm{NC}$, thus providing the driving force for the continuation of the process until either the primary NCs are fully consumed or their number density drops below a critical level which no longer supports a significant collision frequency, or the reaction is forced to halt by a temperature drop (quenching). This results in a discontinuous, stepwise increase of the NC sizes, which passes through a bimodal distribution at 


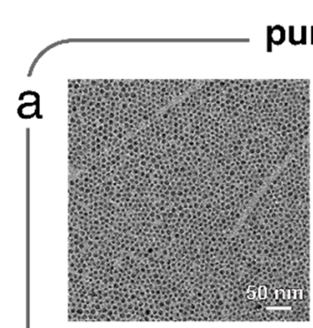

pure Zinc blende

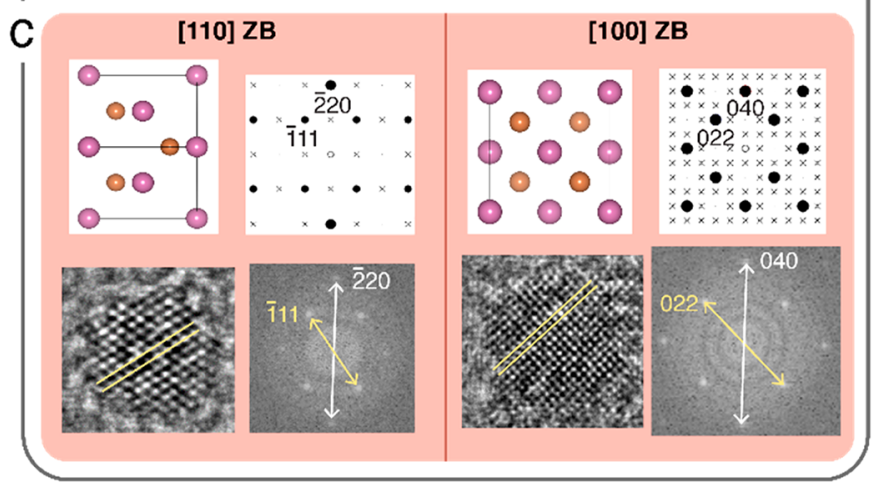

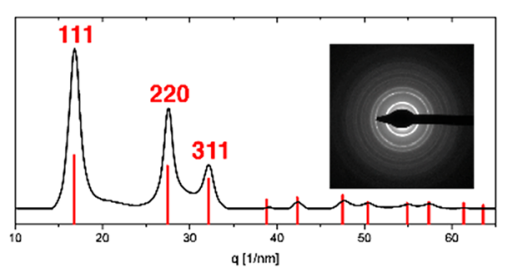

$[100]$ ZB

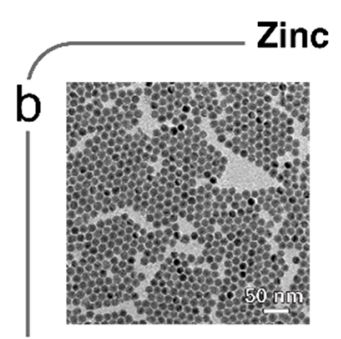

d

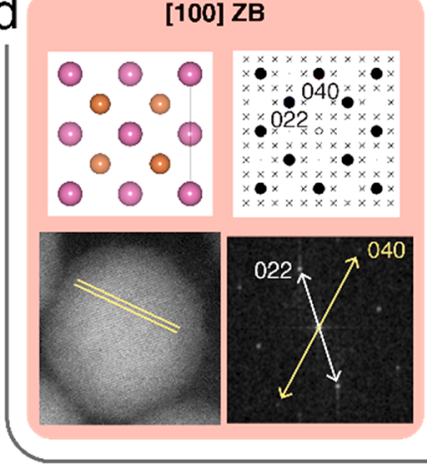

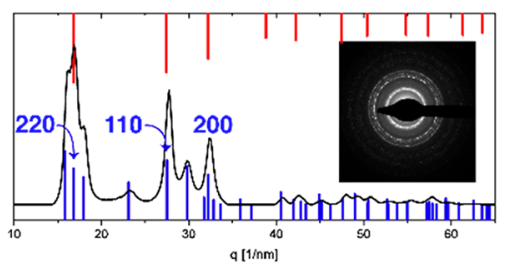

[001] WZ

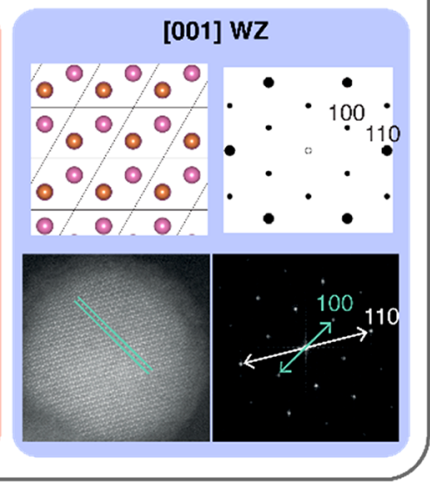

Figure 4. Crystal structure analysis of representative InSb QDs. (a, b) TEM images and corresponding azimuthally integrated ED patterns of InSb QD samples of average diameter (a) $5.3 \pm 1.2 \mathrm{~nm}$ and (b) $14.9 \pm 0.9 \mathrm{~nm}$. Original ED patterns are also included as insets. (c) Representative HRTEM images of InSb QDs from the sample shown in (a) and corresponding FFT. (d) Representative HAADF-STEM images of InSb QDs from the sample shown in (b) and corresponding FFT. For each crystallographic orientation, a schematic of the lattice projection and a simulated single-crystal diffraction pattern (DP) along the given zone axis are shown (for wurtzite the [uvw] notation is used). Selected lattice planes contributing to the pattern are highlighted. Additional HAADF-STEM images of the sample shown in (b), in different crystallographic orientations, as well as the images shown in (d) are reported in full size in the Supporting Information (Figure S20).

intermediate stages when primary and product NCs coexist. Aggregative growth has been often observed in the formation of metal NCs by reduction of metal salts in solution. ${ }^{45-49}$ For example, detailed mechanistic studies of the formation of $\mathrm{Au}$ and Ag NCs by reduction of the metal salts by borohydride,

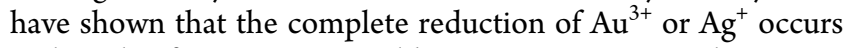
within the first $200 \mathrm{~ms}$, yielding primary NCs with $1.6 \mathrm{~nm}$ diameter in the case of $\mathrm{Au}$ and $2 \mathrm{~nm}$ in the case of $\mathrm{Ag} .{ }^{46,47}$ The primary NCs subsequently grow by aggregation and coalescence to a final diameter of $3.4 \mathrm{~nm}$ for $\mathrm{Au}$ and $4.6 \mathrm{~nm}$ for Ag. ${ }^{46,47}$ Interestingly, in the case of Ag NCs a second aggregation stage starts after a pause of $6-8 \mathrm{~min}$, leading to 12 $\mathrm{nm}$ diameter NCs. ${ }^{47}$ The intermediate pause was attributed to surface stabilization of the $4.6 \mathrm{~nm}$ diameter $\mathrm{Ag} \mathrm{NCs}$ by borohydride coordination. ${ }^{47}$ The borohydride slowly hydrolyzed, ultimately initiating the second growth stage. ${ }^{47}$ This shows that multiple aggregation and coalescence stages are possible under continuously changing physicochemical conditions.

In the present case, we infer that the primary InSb NCs formed shortly after the coreduction of the InSb precursor to $[\mathrm{InSb}]_{n}$ clusters have diameters in the $1-2 \mathrm{~nm}$ range, since NCs in this size range are often isolated by size fractionation of the synthesized InSb QD samples (Figure S18). They are typically recovered as short strings containing a few attached NCs (Figure S18), which is consistent with their presumed role of primary building blocks in the aggregation and coalescence events that ultimately lead to differently sized InSb QDs through multiple sequential stages of aggregative growth. The final NC size at the end of an aggregation and coalescence stage is determined by the NC pair-interaction potentials, which are in turn governed by the balance between attractive potentials (typically van der Waals interactions) that promote aggregation and repulsive potentials (electrostatic or steric hindrance due to ligands) that inhibit aggregation. ${ }^{45,52}$ Consequently, any condition that influences steric, van der Waals or electrostatic interactions between NCs may influence and direct aggregative growth (e.g., ionic strength, surfactants, NC number density, solvent, temperature). ${ }^{52}$ The fact that the final InSb QD mean size and polydispersity were essentially constant after $1 \mathrm{~min}$ reaction implies that the aggregative growth process is fast and is not followed by ripening processes, probably due to the insolubility of $\mathrm{InSb}$ in the reaction mixture, which precludes dissolution and mass transport between smaller and larger NCs in the ensemble. Further, the minor influence of the reaction temperature on the QD sizes can be interpreted as evidence that the pairinteraction potentials are only weakly temperature dependent over the $230-260{ }^{\circ} \mathrm{C}$ range.

On the other hand, the pronounced impact of both the $\mathrm{SH} /$ $\mathrm{InSb}$ ratio and the delay between $\mathrm{SH}$ addition and the injection of the InSb precursor can be rationalized by considering that these variables affect both the coreduction kinetics and the ionic strength of the reaction medium, since $\mathrm{SH}$ is a salt $\left(\mathrm{LiEt}_{3} \mathrm{BH}\right)$. Moreover, it is plausible that, in addition to OLA (and other primary alkylamines), $\mathrm{Et}_{3} \mathrm{BH}^{-}$and the products of its decomposition may also act as weakly coordinating surface ligands. The intriguing effect of the delay between $\mathrm{SH}$ addition and InSb precursor injection is not yet fully understood but cannot be attributed to the mere evaporation of superhydride over time, since this should have the same effect as lower $\mathrm{SH} /$ $\mathrm{InSb}$ ratios (i.e., larger $\mathrm{QD}$ sizes, see Figure S13), in contrast to our observations (viz., bimodal size distributions, see Figure 3 above). We propose that this effect is due to the product of a side reaction between $\mathrm{SH}$ and OLA, although the nature of this side reaction is yet unclear. The role of side reactions involving 
impurities can be excluded since other long-chain primary alkylamines, such as ODA or DDA, give essentially the same results as technical OLA, despite their high purities. One possibility is that at high temperatures $\mathrm{SH}$ reacts with primary amines (or their protonated ammonium form) to produce aminoborohydrides. Lithium aminoborohydrides are produced by reaction between $n$-butyllithium and amine-borane complexes $\mathrm{H}_{3} \mathrm{~B} / \mathrm{NHR}_{2}$ at $0{ }^{\circ} \mathrm{C} .{ }^{55}$ Although analogous reactions between $\mathrm{SH}$ and primary amines at high temperatures have never been investigated, we deem it possible that they would occur, even if to a limited extent. Considering that aminoborohydrides are known to be powerful, but more stable, reducing agents, ${ }^{55}$ their presence in the reaction medium would certainly influence the reduction kinetics of the injected InSb precursors. This would explain why InSb QDs prepared with short $\mathrm{SH}-\mathrm{InSb}$ delays are typically accompanied by a small fraction of nonstoichiometric nanocrystals (see Figure S14 and discussion above). The presence of different reducing species in the reaction medium after long $\mathrm{SH}-\mathrm{InSb}$ delays could also affect the outcome of the synthesis by modulating the ionic strength of the medium and/or the coordinating ability of borohydride species.

Colloidal InSb QDs: Size and Crystal Structure. As mentioned above, the synthesis procedure developed in our work yields $\mathrm{InSb} \mathrm{QD}$ ensembles with diameters ranging from $2.8 \pm 0.3$ to $18.2 \pm 1.6 \mathrm{~nm}$ after size-selective precipitation (Figure 2 above) (see the Methods for details concerning the size fractionation procedure). The elemental composition of the synthesized InSb QDs was investigated by energy dispersive X-ray spectroscopy (EDS) and found to be very close to the expected stoichiometry $(\mathrm{In} / \mathrm{Sb}=1: 1.10 \pm 0.03$, regardless of the QD size). Additionally, indium and antimony appear to be homogeneously distributed (Figure S19). Electron diffraction (ED) patterns of QD ensembles as well as high-resolution TEM (HRTEM) and high angle annular dark field scanning transmission electron microscopy (HAADF-STEM) images of single QDs were used to assign a zinc blende ( $\mathrm{ZB})$ crystal structure to InSb QDs with diameters in the $2.8 \pm 0.3$ to $6.0 \pm 0.7 \mathrm{~nm}$ range (Figure $4 \mathrm{a}, \mathrm{c}$ ). On the other hand, ED patterns of $\mathrm{QD}$ ensembles with diameters in the $10.3 \pm 1.2$ to $18.2 \pm 1.6 \mathrm{~nm}$ range revealed the coexistence of $\mathrm{ZB}$ and wurtzite (WZ) crystal structures (Figure 4b,d).

Figure 4a,b presents the azimuthally integrated ED patterns of InSb QD samples of two different diameters (5.3 $\pm 1.2 \mathrm{~nm}$ and $14.9 \pm 0.9 \mathrm{~nm}$, respectively). The diffractograms of $\mathrm{WZ}$ and $\mathrm{ZB}$ show many peaks in common since the two structures differ only in the stacking arrangement of the close-packed layers. In particular, the first three most intense scattering peaks of the ZB phase, corresponding to the (111), (220), and (311) lattice planes, occur at almost the same $q$-values as the (002), (110) and (200) reflections of the WZ phase, respectively (Figure 4a,b). However, the latter structure has additional reflections in the same q-region due to its lower symmetry. The relative intensities of the peaks cannot be interpreted quantitatively, since the QDs are not necessarily randomly oriented on the TEM grid, but often assume preferential orientations due to their specific faceting. This means that, while a pure $\mathrm{ZB}$ phase can be unambiguously ascribed to the $5.3 \mathrm{~nm}$ sample (Figure $4 \mathrm{a}$ ) due to the absence of reflections corresponding to the $\mathrm{WZ}$ phase, the distinction between a pure $\mathrm{WZ}$ phase and a WZ-ZB polymorph solely on the basis of the ensemble ED pattern of the $14.9 \mathrm{~nm}$ sample
(Figure $4 \mathrm{~b}$ ) is challenging, since the observation of the characteristic reflections of the $\mathrm{WZ}$ phase confirm its presence, but cannot exclude coexistence with $\mathrm{ZB}$.

This ambiguity can be resolved by analyzing HRTEM images of single InSb QDs. The fast Fourier transform (FFT) of the HRTEM images can be indexed according to the simulated single-crystal diffraction patterns (DP) along the same zone axis. Two representative HRTEM images of $\mathrm{ZB}$ $\mathrm{InSb} \mathrm{QDs}$ from the sample shown in Figure 4a $(5.3 \mathrm{~nm}$ diameter) are reported in Figure 4c, and their FFT patterns are indexed according to the simulated DP along the [110] and [100] zone axis of the $\mathrm{ZB}$ crystal structure. The lattice planes contributing to the pattern (those with the normal perpendicular to the zone axes) are also shown. The same analysis performed on HAADF-STEM images of single $\mathrm{InSb}$ QDs from the sample shown in Figure $4 \mathrm{~b}$ (14.9 nm diameter) reveal the coexistence of projections that can be unambiguously assigned to either $\mathrm{WZ}$ or $\mathrm{ZB}$, depending on the $\mathrm{QD}$ (Figure $4 \mathrm{~d}$ ). This shows that ensembles of large (viz. 10.3 to $18.2 \mathrm{~nm}$ diameter) InSb QDs consist of a mixture of $\mathrm{WZ}$ and $\mathrm{ZB}$ QDs (Figure S20). ZB is the thermodynamically stable crystal structure of bulk InSb at room temperature, while $\mathrm{WZ}$ is metastable. Nanoscale InSb most commonly adopts the ZB structure, especially for colloidally synthesized NCs. ${ }^{22,24}$ However, colloidal InSb QDs exhibiting ZB-WZ polymorphism have also been reported, ${ }^{23}$ as well as polytypic InSb nanowires consisting of twinning single crystalline superlattices of alternating $\mathrm{ZB}$ and $\mathrm{WZ}$ domains. ${ }^{56} \mathrm{ZB}-\mathrm{WZ}$ polymorphism is well-documented for colloidal NCs of II-VI (e.g., CdSe $)^{40}$ and $\mathrm{I}-\mathrm{III}-\mathrm{VI}_{2}\left(\text { e.g., } \mathrm{CuInS}_{2}\right)^{44}$ semiconductors and is rationalized by considering the small energy differences between the two crystal structures. Many factors can contribute to stabilize metastable crystal structures at the nanoscale, such as temperature and growth conditions as well as the coordinating ability of ligands toward specific crystallographic facets. ${ }^{40}$

Optical Properties of Colloidal InSb QDs. The nearinfrared absorption and photoluminescence (PL) spectra of representative $\mathrm{InSb}$ QDs with diameter ranging from $2.9 \pm 0.3$ to $4.2 \pm 0.5 \mathrm{~nm}$ are shown in Figure 5. Additional absorption spectra for InSb QD ensembles in the $2.8 \pm 0.3 \mathrm{~nm}$ to $6.0 \pm$ $0.7 \mathrm{~nm}$ diameter range are presented in Figure 6a. The absorption spectra do not show sharp first absorption peaks (Figures 5 and 6a). This can be ascribed to the fact that the ensemble size polydispersity of the investigated InSb QDs is in the $10-12 \%$ range. Considering that these QDs are strongly quantum confined $\left(r_{\mathrm{QD}} \leq 0.05 a_{0}\right.$, with $\left.a_{0}=61 \mathrm{~nm}\right),{ }^{1}$ even relatively small size deviations will result in large shifts in the exciton transition energies, contributing to the broadening of the spectral features. In the absence of a distinct absorption peak, a reliable method to extract the peak position of the lowest energy absorption transition $\left(E_{1}\right)$ of the $\operatorname{InSb} \mathrm{QD}$ samples is by locating the minimum of the second derivative of their absorption spectra. This analysis shows that the $E_{1}$ values of the InSb QD ensembles prepared in this work shift from $0.79 \mathrm{eV}(1570 \mathrm{~nm})$ to $1.28 \mathrm{eV}(969 \mathrm{~nm})$ as the average QD diameter decreases from 6.0 to $2.8 \mathrm{~nm}$ (Figure 6a). The size dependence of the lowest energy absorption transition will be discussed in more detail below.

The PL peak energies are also strongly size dependent, shifting from 0.882 to $1.164 \mathrm{eV}$ as the InSb QD diameter decreases from 4.2 to $2.9 \mathrm{~nm}$ (Figure 5 and Table S2). The full-width at half-maximum (fwhm) of the PL peaks is sizeindependent (viz. $132 \pm 8 \mathrm{meV}$, Table S2). The PL is clearly 


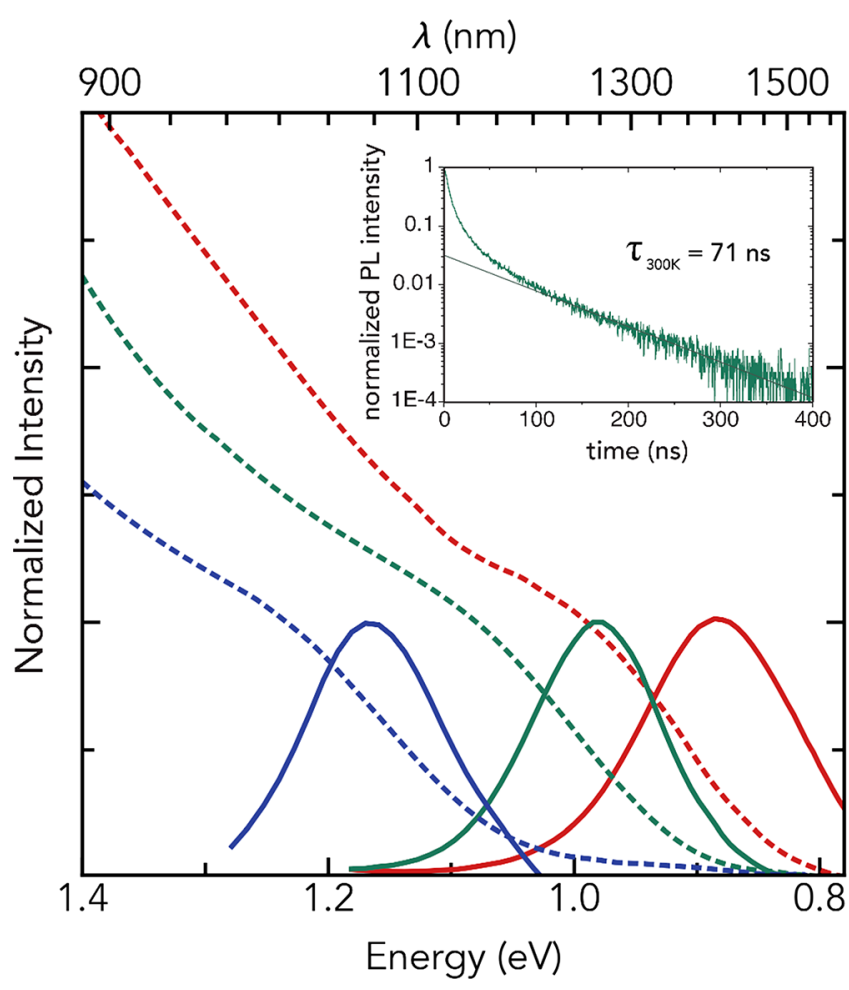

Figure 5. Absorption (dashed lines) and photoluminescence (solid lines) spectra of colloidal suspensions of $2.9 \pm 0.3$ (blue), $3.7 \pm$ 0.5 (green), and $4.2 \pm 0.5$ (red) nm diameter InSb QDs in toluene. Inset: Photoluminescence decay trace of a colloidal suspension of $3.7 \pm 0.5 \mathrm{~nm}$ diameter InSb QDs in toluene. The solid line is a single-exponential fit. The measurements were carried out at room temperature.

due to band-edge recombination since the (nonresonant) Stokes shift is small (70 to $\sim 90 \mathrm{meV}$, Table S2). The contribution of trap-assisted radiative recombination to the PL transition can also be excluded on the basis of the roomtemperature PL decay transients (see Figure 5, inset, for a representative example), which consist of a fast multiexponential decay component (ascribed to QDs in which nonradiative recombination dominates) followed by a slow single-exponential component with a decay constant of $71 \mathrm{~ns}$ for $3.7 \pm 0.5 \mathrm{~nm} \mathrm{InSb}$ QDs (170 ns at $4 \mathrm{~K}$, Figure S21). This slow component is ascribed to the exciton radiative lifetime and is similar to that previously reported by Talapin and coworkers for $\sim 4.5 \mathrm{~nm}$ diameter InSb QDs with PL peak at 1500 $\mathrm{nm}($ viz. $\sim 160 \mathrm{~ns}))^{22}$ Such a short exciton radiative lifetime (for reference, the radiative lifetime of PbSe QDs emitting in the same spectral range is $\sim 3 \mu \mathrm{s})^{57}$ not only excludes the possibility that the observed PL is due to trap-assisted radiative recombination, but also strongly supports the notion that the direct nature of the bandgap of bulk InSb is preserved in $\mathrm{InSb}$ QDs. The relevance of this observation will be discussed in more detail below.

As discussed above, the absorption and PL transitions of the colloidal InSb QDs prepared in our work shift to higher energies with decreasing $\mathrm{QD}$ size (Figures 5 and $6 \mathrm{a}$ ), as expected considering that they are in the strong quantum confinement regime. To quantitatively analyze the size dependence of the band gap of InSb QDs we constructed a sizing curve correlating the peak position of the lowest energy absorption transition $\left(E_{1}\right)$ (estimated from the absorption spectra) with the QD diameters determined from TEM images (Figure $6 \mathrm{~b}$, solid circles). For comparison, experimental data (Figure 6b, solid blue symbols) ${ }^{22-24}$ and theoretically calculated values (Figure $6 \mathrm{~b}$, empty symbols) ${ }^{16,58,59}$ from previous works are also included. The size dependence observed in the data sets from different experimental works is similar, despite some systematic differences. These discrepancies can be ascribed to the inherent experimental uncertainties associated with the accurate determination of mean diameters from TEM images and mean band gap energies from absorption spectra of ensembles of polydisperse colloidal QDs, as recognized in previous works dealing with the construction of empirical sizing curves of a variety of QD compositions ( $\mathrm{CdSe}, \mathrm{CdTe}$, InAs, $\mathrm{PbS}, \mathrm{PbSe}$, and $\left.\mathrm{CuInS}_{2}\right) \cdot{ }^{60-65}$ In the case of InSb QDs, an oxide shell may be present leading to overestimation of the sizes obtained from TEM images. When this is the case, the diameters must be corrected by subtraction of the shell thickness $(1.2 \mathrm{~nm})$, as has been done by Yarema et $a l^{23}$ (Figure $6 \mathrm{~b}$ presents both the observed and the corrected diameters reported in ref 23). This correction is, however, not necessary for the QD diameters reported in the present work since the presence of an oxide layer can be excluded based on HRTEM images. The experimental data reported by Liu et al. ${ }^{22}$ and Zhao et al. ${ }^{24}$ are also not affected by the presence of an oxide shell.

It is interesting to note that the size dependence of the band gap of colloidal InSb QDs observed in all of the experimental data sets collected in Figure $6 \mathrm{~b}$ is much weaker than the $\mathrm{E} \propto$ $1 / d^{2}$ trend predicted using the theoretical model proposed by Efros and Rosen, ${ }^{58}$ which is based on an eight-band effectivemass approximation (EMA) approach that simultaneously takes into account the nonparabolicity of the electron- and light-hole dispersion and the coupling between conduction and valence bands. The systematic deviation between the experimentally observed band gaps and those theoretically predicted on the basis of the EMA model ${ }^{58}$ has already been noticed by Talapin and co-workers ${ }^{22}$ and attributed to two possible reasons: (i) leakage of the exciton wave function into the surrounding medium due to the finite potential offset between the QD and the ligand layer ${ }^{22,66}$ and (ii) transition from direct to indirect bandgap for sufficiently small InSb QDs $(d<5 \mathrm{~nm})$ due to the different electron effective masses at the $\Gamma$ - and L-points (viz. $0.0139 \mathrm{~m}_{0}$ and $0.25 \mathrm{~m}_{0}$, respectively), ${ }^{1}$ which should lead to a much faster energy shift of the $1 S$ level at the $\Gamma$-point than at the $L$-point with reduction of the $Q D$ size, so that the L-point would eventually become lower in energy than the $\Gamma$-point. ${ }^{22,67-69}$ However, we note that the latter hypothesis is inconsistent with the short exciton radiative lifetimes reported for both $3.7 \pm 0.5 \mathrm{~nm}$ (71 ns at room temperature and $170 \mathrm{~ns}$ at $4 \mathrm{~K}$, Figures 5 and S21, respectively) and $\sim 4.5 \mathrm{~nm}$ diameter $(\sim 160 \mathrm{~ns})^{22} \mathrm{InSb}$ QDs. Moreover, other theoretical approaches, based on either atomistic tight-binding ${ }^{59}$ or semiempirical pseudopotential (SE-PP) methods, $^{16}$ yield band gap values that are much more in line with the experimental data, although still overestimating the strength of the size-dependence. The only exception seems to be the theoretical calculations done by using the SE-PP method for Sb-centered InSb QDs (Sb-rich for $d<6 \mathrm{~nm}),{ }^{16}$ which follow a weaker size-dependent trend (Figure 6b).

To facilitate the quantitative comparison between the empirical and theoretical size-dependent trends, we fitted our 

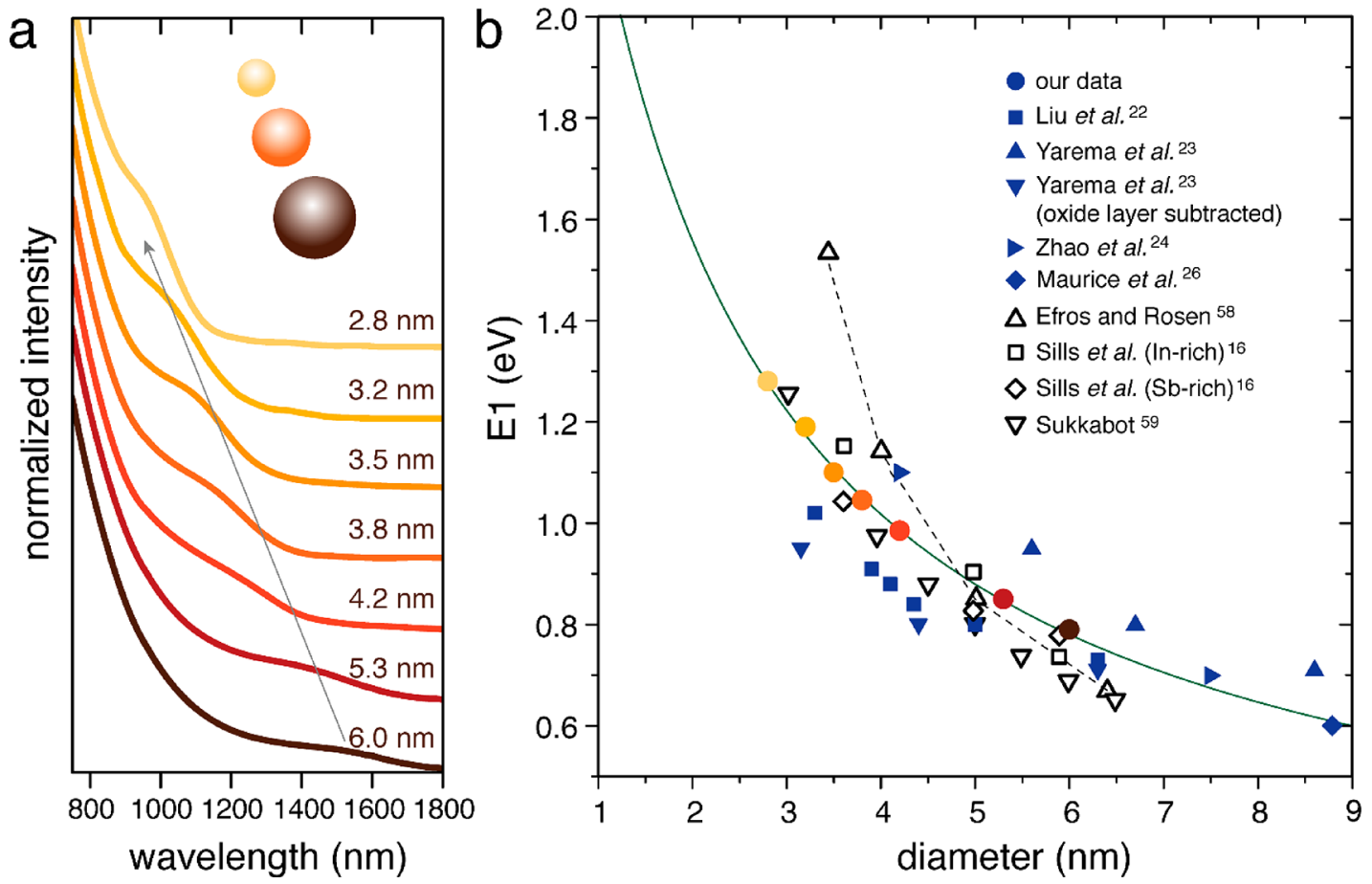

Figure 6. (a) Near-infrared absorption spectra of colloidal suspensions of InSb QDs with diameters ranging from $2.8 \pm 0.3$ to $6.0 \pm 0.7 \mathrm{~nm}$. The ensemble size polydispersity is $\sim 10 \%$ for all samples. The arrow highlights the shift of the lowest energy exciton absorption transition (i.e., the band gap) to higher energies with decreasing QD diameter. (b) Size dependence of the band gap ( $\left.E_{1}\right)$ of colloidal InSb QDs showing our own experimental data (solid circles, color code is the same used in panel a), as well as experimental data from previous works (Liu et al. $\sim 5 \%$ polydispersity, ${ }^{22}$ Yarema et al. $\sim 15 \%$ polydispersity, ${ }^{23}$ Zhao et al. $\sim 10 \%$ polydispersity, ${ }^{24}$ and Maurice et al. $\sim 13 \%$ polydispersity, ${ }^{26}$ solid blue symbols) and theoretically calculated values reported by Efros and Rosen (eight-band effective-mass approximation approach), ${ }^{58}$ Sills et al. (semiempirical pseudopotential approach), ${ }^{16}$ and Sukkabot (atomistic tight-binding approach) ${ }^{59}$ (empty symbols). The green solid line is a fit to our experimental data based on eq 2 above.

experimental data to an expression previously proposed by Allan and Delerue based on tight-binding calculations ${ }^{70}$

$$
E_{\mathrm{g}}(d)=E_{\mathrm{g}}(\infty)+\frac{1}{a d^{2}+b d+c}
$$

where $E_{g}(\mathrm{~d})$ and $E_{\mathrm{g}}(\infty)$ are the band gap (in $\mathrm{eV}$ ) for a QD of diameter $d$ (in $\mathrm{nm}$ ) and for bulk, respectively, and $a, b$, and $c$ are constants. Equation 1 has been successfully used to fit the experimentally observed size-dependent trends for $\mathrm{PbSe},{ }^{62,70}$ $\mathrm{PbS},{ }^{71} \mathrm{CdSe},{ }^{63} \mathrm{CdTe},{ }^{63}$ and $\mathrm{CuInS}_{2}{ }^{65}$ QDs. Fitting our experimental data for InSb QDs (solid circles in Figure $6 \mathrm{~b}$ ) to eq 1 without any constraints (except that $a, b$, and $c \geq 0$ ) yields $\left(\chi^{2} \sim 0.996\right)$

$$
E_{\mathrm{g}}(d)=0.17+\frac{1}{0.2295 d+0.2618}
$$

Equation 2 clearly shows that the empirical size dependence of the band gap of InSb QDs is entirely determined by the $1 / d$ term. This is in striking contrast with the $1 / d^{2}$ size dependence predicted by theoretical models based on the effective mass approximation (dashed black line in Figure $6 \mathrm{~b}) .{ }^{58}$ The $1 / d^{2}$ term is typically associated with the quantum confinement contribution as described within the parabolic effective mass approximation, while the $1 / d$ term reflects the electron-hole Coulomb interaction. ${ }^{58,70}$ The contribution of the Coulomb interaction is often taken to be negligible, since it decreases with increasing dielectric constant $\varepsilon$ due to dielectric screening. This is indeed the case for QDs of polar semiconductors such as $\mathrm{CdSe}$ and $\mathrm{CdTe}\left(\varepsilon=8\right.$ and 12 , respectively), ${ }^{63}$ which exhibit band gaps following a size-dependence that is almost entirely due to the $1 / d^{2}$ term. ${ }^{63}$ Considering that the dielectric constant of bulk InSb (viz., 16.8) ${ }^{1}$ is even larger than those of $\mathrm{CdSe}$ and $\mathrm{CdTe}$ one would expect Coulomb interaction contributions to be even less relevant for InSb QDs. Nevertheless, a $1 / d$ band gap size-dependence has been reported for QDs of several different semiconductors, despite their high dielectric constants (viz., $\mathrm{PbSe},{ }^{62,70} \mathrm{PbS},{ }^{71} \mathrm{CuInS}_{2},{ }^{65}$ and InP, ${ }^{72,73}$ with bulk $\varepsilon=23,^{74} 17,,^{74} 10,{ }^{65}$ and $12.5,^{75}$ respectively).

The dominance of the $1 / d$ term in the size dependence of the band gap of $\mathrm{Pb}$-chalcogenide QDs has been interpreted as evidence that the energy dispersion of the conduction and valence bands around the band gap is mostly linear, consistent with theoretical tight-binding calculations that show the bands to be parabolic only in a very small region near the maximum of the valence band and minimum of the conduction band. ${ }^{70}$ Our observation that the size-dependence of the band gap of InSb QDs follows a $1 / d$ dependence thus implies that the nonparabolicity of the electron- and light-hole dispersion is even more pronounced than assumed by Efros and Rosen in their model. ${ }^{58}$ Moreover, one should also consider that, as pointed out by Efros and Rosen, ${ }^{58}$ the electron-hole Coulomb interaction in small QDs is not described by the static dielectric constant, but rather by the high frequency dielectric constant, which is strongly dependent on the electronic polarizability. As a result, the effective dielectric constant of a QD may be considerably smaller than that of the corresponding bulk material. We note that this effect is likely even more pronounced in covalent semiconductors, consistent with the fact that the materials showing a $1 / d$ dependence are 
all covalent in nature. From this perspective, it is interesting to notice that semiempirical pseudopotential calculations for $\mathrm{Sb}$ centered $\mathrm{InSb} \mathrm{QDs}^{16}$ yield band gap values and a size dependent trend that are very close to the experimental data (Figure 6b). This has been attributed by Sills et al. ${ }^{16}$ to the slike symmetry of the valence band maximum in both In-rich and Sb-rich QDs, and admixture of $\Gamma$-point and L-point $k$ vectors of the conduction band minimum in Sb-rich QDs.

Equation 2 is also valuable as a sizing curve, allowing one to estimate the average diameter of InSb QDs from the peak position of their lowest energy absorption transition. For example, the diameter of the small InSb QDs reported by Crisp et al., ${ }^{27}$ showing the first absorption transition at $\sim 650 \mathrm{~nm}$ $(\sim 1.91 \mathrm{eV})$, is estimated with eq 2 to be $1.4 \mathrm{~nm}$, which is in good agreement with the $\sim 1 \mathrm{~nm}$ diameter estimated in ref 27 from TEM images. Further, the agreement between the size estimated using eq 2 and that experimentally determined by Maurice et $a l^{26}$ for the InSb QDs prepared in their work is excellent (see Figure 6). For the $[\mathrm{InSb}]_{n}$ clusters that form in the reaction mixture a few seconds after the injection of the $\mathrm{InSb}$ precursor solution (see Synthesis of Colloidal InSb QDs for a detailed discussion), an effective diameter of $0.7 \mathrm{~nm}$ can be estimated from their lowest energy absorption peak at 370 $\mathrm{nm}(3.35 \mathrm{eV}$, Figure S12). Although the concept of "diameter" is no longer meaningful in the cluster size regime, the "effective diameter" obtained from eq 2 is quite insightful as it allows us to estimate that the number $n$ of InSb units in the clusters is $\sim 2.6$ by considering the bulk density of $\operatorname{InSb}\left(5.78 \mathrm{~g} / \mathrm{cm}^{3}\right)$ and the cluster volume. As discussed above (Synthesis of Colloidal InSb QDs), this estimated $n$ supports our hypothesis that at sufficiently high temperatures the reduction kinetics of both In and $\mathrm{Sb}$ are sufficiently fast to convert the InSb precursor complex(es) directly to $[\mathrm{InSb}]_{n}$ molecular clusters.

\section{CONCLUSION}

In summary, our work shows that Lewis acid-base interactions between $\mathrm{Sb}$ (III) and $\mathrm{In}$ (III) complexes formed in situ from commercially available compounds (viz., $\mathrm{InCl}_{3}, \mathrm{Sb}\left[\mathrm{NMe}_{2}\right]_{3}$ and a primary alkylamine) lead to the formation of amino and amido complexes containing $\mathrm{Sb}-\mathrm{In}$ bonds. We demonstrate that these complexes can be used as single source precursors for the synthesis of colloidal InSb QDs ranging from 2.8 to $18.2 \mathrm{~nm}$ in diameter by fast coreduction with $\mathrm{LiEt}_{3} \mathrm{BH}$ in oleylamine (or other high boiling point alkylamine) at sufficiently high temperatures $\left(\geq 230^{\circ} \mathrm{C}\right)$. The most effective ways to tailor the size of the QDs are the molar ratio between the amount of superhydride and $\mathrm{InSb}$ and the time delay between the addition of superhydride to oleylamine at $240{ }^{\circ} \mathrm{C}$ and the injection of the InSb precursor solution. Our findings allow us to propose a formation mechanism for the colloidal InSb QDs synthesized in our work, which is based on a nonclassical nucleation event (formation of $[\mathrm{InSb}]_{n}$ clusters by fast reduction of the InSb precursors), followed by growth by aggregation and coalescence. The aggregative growth yields ensembles with multimodal size distributions, which can be fractionated in subensembles with relatively narrow polydispersity $(\leq 10 \%)$ by postsynthetic size-selective precipitation. $\mathrm{InSb}$ QDs with diameters in the 2.8 to $6.0 \mathrm{~nm}$ range have the zinc blende crystal structure, while ensembles of larger (viz., 10 to $16 \mathrm{~nm}$ diameter) InSb QDs consist of a mixture of wurtzite and zinc blende QDs. Finally, we constructed a sizing curve correlating the peak position of the lowest energy absorption transition $\left(E_{1}\right)$ with the QD diameters, which shows that the band gap of colloidal InSb QDs increases with size reduction following a $1 / d$ dependence. The small Stokes shifts and the short exciton radiative lifetime show that the PL is due to band-edge recombination and imply that the direct nature of the bandgap of bulk InSb is preserved in InSb QDs.

\section{METHODS}

Materials. The materials used were oleylamine (OLA, SigmaAldrich, 70\%), octadecylamine (ODA, Sigma-Aldrich, $\geq 99.0 \%$ ), hexadecylamine (HDA, Sigma-Aldrich, 98\%), dodecylamine (DDA, Sigma-Aldrich, $\geq 99.0 \%$ ), octylamine (Sigma-Aldrich, 97\%), toluene (anhydrous, Sigma-Aldrich, 99.8\%), tris(dimethylamido)antimony(III) $\left(\mathrm{Sb}\left[\mathrm{NMe}_{2}\right]_{3}\right.$, Sigma-Aldrich, 99.99\%), indium(III)chloride $\left(\mathrm{InCl}_{3}\right.$, Sigma-Aldrich, 98\%), lithium triethylborohydride $\left(\mathrm{LiEt}_{3} \mathrm{BH}\right.$, Super-Hydride, Sigma-Aldrich, $1 \mathrm{M}$ in tetrahydrofuran, THF), dioctyl ether (DOE, Alfa Aesar, 99\%), 1-butanol (anhydrous, Sigma-Aldrich, 99.8\%), methanol (anhydrous, Sigma-Aldrich, 99.8\%), methyl acetate (MeOAc, anhydrous, Sigma-Aldrich, 99.5\%), and oleic acid (SigmaAldrich, 90\%). All chemicals were used as received except for oleylamine and oleic acid, which were degassed before use. The degassing was performed at $100{ }^{\circ} \mathrm{C}$ under reduced pressure $(\sim 1$ mbar) for $4 \mathrm{~h}$.

Preparation of Superhydride Solution. The solvent of the $\mathrm{LiEt}_{3} \mathrm{BH}$ solution was exchanged for DOE by mixing equal volumes of $\mathrm{DOE}$ and $\mathrm{LiEt}_{3} \mathrm{BH}$ in THF and degassing the mixture at room temperature in a Schlenk line under reduced pressure $(\sim 1 \mathrm{mbar})$ until the volume of the solution was reduced by half. The final $\mathrm{LiEt}_{3} \mathrm{BH}$ solution has a concentration of $1.0 \mathrm{M}$ in DOE. Caution: $\mathrm{LiEt}_{3} \mathrm{BH}$ is very reactive in air and should be handled using air-free procedures.

Preparation of the InSb Precursor Solution. Toluene $(2 \mathrm{~mL})$, $0.497 \mathrm{~g}$ of OLA $(1.86 \mathrm{mmol})$, and $0.200 \mathrm{~g}$ of $\mathrm{Sb}\left[\mathrm{NMe}_{2}\right]_{3}(0.78 \mathrm{mmol})$ were mixed together in a glovebox under $\mathrm{N}_{2}\left(\mathrm{H}_{2} \mathrm{O}\right.$ and $\left.\mathrm{O}_{2}<2 \mathrm{ppm}\right)$ yielding a bright yellow solution. $\mathrm{InCl}_{3}(0.173 \mathrm{~g}, 0.78 \mathrm{mmol})$ was added and dissolved under stirring at room temperature. The resulting clear, bright yellow solution is stable for at least several weeks under $\mathrm{N}_{2}$.

Synthesis of Colloidal InSb Quantum Dots. In a typical synthesis, $10 \mathrm{~mL}$ of degassed OLA was heated to $240{ }^{\circ} \mathrm{C}$ in a roundbottom flask under constant stirring. At this temperature, $1.4 \mathrm{~mL}$ of $\mathrm{LiEt}_{3} \mathrm{BH}$ in DOE or THF $(1.0 \mathrm{M}, 1.2 \mathrm{mmol})$ was added in a dropwise manner. During the addition, OLA acquired an orange color. After the addition, the temperature was kept steady at $240{ }^{\circ} \mathrm{C}$ for a duration of 2-10 min. Subsequently, $0.25 \mathrm{~mL}$ of the precursor solution was injected using a Finn pipet. After the temperature was kept at $240{ }^{\circ} \mathrm{C}$ for 2-5 min, the reaction solution was transferred to a vial containing $6 \mathrm{~mL}$ of anhydrous toluene using a glass syringe. Oleic acid $(1 \mathrm{~mL})$ was added to the vial, $2 \mathrm{~min}$ after quenching the reaction into toluene. The solution was kept at room temperature for $30 \mathrm{~min}$. The diluted reaction mixture was separated in six portions, and to each of them 3 $\mathrm{mL}$ of $\mathrm{BuOH}$ and $3 \mathrm{~mL}$ of $\mathrm{MeOH}$ were added, leading to turbidity. Centrifugation for $10 \mathrm{~min}$ at $2800 \mathrm{rpm}$ yielded a black precipitate and colorless supernatant. The colorless supernatant was discarded, and the black precipitate was redispersed in $1 \mathrm{~mL}$ of toluene or tetrachloroethylene (TCE) to yield stable suspensions. The purification process was repeated once. All of the synthesis and purification steps were carried out in an inert atmosphere under anhydrous conditions $\left(\mathrm{N}_{2}\right.$ glovebox, $\mathrm{H}_{2} \mathrm{O}$ and $\left.\mathrm{O}_{2}<2 \mathrm{ppm}\right)$. Detailed synthesis protocols for a few selected $\mathrm{QD}$ sizes are provided in the Supporting Information (section S5 and Figure S22).

Size-Selective Precipitation. To the purified samples dispersed in toluene was added a small amount of $\mathrm{MeOAc}$, after which the sample was centrifuged for $10 \mathrm{~min}$ at $2800 \mathrm{rpm}$ and the precipitate was separated from the supernatant and redispersed in toluene. This cycle of (1) addition of small amounts of MeOAc to the supernatant, (2) centrifugation, and (3) separation of the precipitate was repeated until the supernatant had become colorless (Figure S22). Typically, to separate $\sim 10$ aliquots from $1 \mathrm{~mL}$ of purified QD dispersion, $\sim 0.4 \mathrm{~mL}$ $\mathrm{MeOAc}$ was needed in the first cycles, and $\sim 1.0-5.0 \mathrm{~mL} \mathrm{MeOAc}$ was needed in the later cycles. 
${ }^{1} \mathrm{H}$ and ${ }^{13} \mathrm{C}$ Nuclear Magnetic Resonance (NMR) spectroscopy. A small amount of the compound under study was dissolved in $0.5 \mathrm{~mL}$ of deuterated toluene so that the resulting solution had a concentration of around $30 \mathrm{mg} / \mathrm{L}$. This solution was transferred to an air-sealed NMR sample tube. The ${ }^{1} \mathrm{H}$ and ${ }^{13} \mathrm{C}$ NMR measurements were performed using an Agilent MRF400 equipped with an NMR probe and Optima Tune system. The spectra were recorded at room temperature using a magnetic field oscillating at $400 \mathrm{MHz}$ and a pulse repetition time of $25 \mathrm{~s}$.

Transmission Electron Microscopy (TEM), Energy Dispersive X-ray Spectroscopy (EDS), and Electron Diffraction (ED). Samples for TEM imaging were prepared by drop-casting a toluene solution of purified NCs onto a carbon-coated 200 mesh copper TEM grid. Conventional TEM images were acquired using either a FEI Tecnai-20FEG microscope or a Thermo Fisher Scientific Talos F200X microscope, both operating at $200 \mathrm{kV}$. Quantum dot sizes were measured from the TEM images by measuring around 80 particles by hand using ImageJ software. HRTEM and EDS measurements were performed on a Thermo Fisher Scientific Talos F200X microscope operating at $200 \mathrm{kV}$ and an aberration corrected "cubed" Thermo Fisher Scientific Titan 60-300 microscope operating at $300 \mathrm{kV}$. The lattice spacings of NCs were obtained byFourier transform from the area of interest in HRTEM images. High angle annular dark field scanning transmission electron microscopy (HAADF-STEM) images were acquired on an aberration corrected "cubed" Thermo Fisher Scientific Titan 60-300 microscope operating at $300 \mathrm{kV}$. For the crystal structure analysis CrystalMaker 9 and SingleCrystal 2 were used.

Optical Spectroscopy. Samples for optical measurements were prepared bydispersing InSb QDs into $3 \mathrm{~mL}$ of anhydrous toluene or tetrachloroethylene (TCE) in sealable quartz cuvettes under $\mathrm{N}_{2}$. Absorption spectra were recorded on a double-beam PerkinElmer Lambda $950 \mathrm{UV} /$ vis/NIR spectrometer $(175-3300 \mathrm{~nm})$. Steady state PL measurements were carried out using an Edinburgh Instruments FLS920 spectrofluorometer equipped with a $900 \mathrm{~W}$ Xe lamp as excitation source and double-grating monochromators for both the excitation and the emission. A liquid $\mathrm{N}_{2}$-cooled Hamamatsu R550972 photomultiplier tube $(750-1600 \mathrm{~nm})$ was used as detector for obtaining PL spectra. The excitation wavelength was $600 \mathrm{~nm}$ and an $830 \mathrm{~nm}$ long pass filter was placed before the detector. The spectra were corrected for the instrumental response. For transient PL measurements, the samples were excited using a Laser-export Co. Ltd., frequency tripled, pulsed Nd:YAG laser at $3.49 \mathrm{eV}(355 \mathrm{~nm})$ with $1 \mathrm{kHz}$ repetition rate and detected using a Oriel Instrument Cornerstone $1 / 4 \mathrm{~m}$ monochromator coupled with a Hamamatsu R5509 NIR photomultiplier tube cooled at liquid nitrogen temperature with a Products for Research, Inc. PC176TSCE005 cooling chamber.

\section{ASSOCIATED CONTENT}

\section{(s) Supporting Information}

The Supporting Information is available free of charge at https://pubs.acs.org/doi/10.1021/acsnano.0c04744.

Additional NMR spectra of the precursor compounds, additional TEM images of the reaction products under various reaction conditions, absorption spectra of early stage reaction aliquots, elemental maps of selected InSb QD samples, high-resolution TEM images of $15 \mathrm{~nm}$ diameter InSb QDs, PL decay curve of $3.7 \mathrm{~nm}$ diameter InSb QDs at $4 \mathrm{~K}$, synthesis details for selected QD sizes (PDF)

\section{AUTHOR INFORMATION}

\section{Corresponding Author}

Celso de Mello Donega - Condensed Matter and Interfaces, Debye Institute for Nanomaterials Science, Utrecht University,
3508 TA Utrecht, The Netherlands; 이이이.org/0000-00024403-3627; Email: c.demello-donega@uu.nl

\section{Authors}

Serena Busatto - Condensed Matter and Interfaces, Debye Institute for Nanomaterials Science, Utrecht University, 3508 TA Utrecht, The Netherlands

Mariska de Ruiter - Condensed Matter and Interfaces, Debye Institute for Nanomaterials Science, Utrecht University, 3508 TA Utrecht, The Netherlands

Johann T. B. H. Jastrzebski - Organic Chemistry and Catalysis, Debye Institute for Nanomaterials Science, Utrecht University, 3584 CG Utrecht, The Netherlands

Wiebke Albrecht - Electron Microscopy for Materials Science (EMAT), University of Antwerp, B-2020 Antwerp, Belgium

Valerio Pinchetti - Dipartimento di Scienza dei Materiali, Università degli Studi di Milano Bicocca, I-20125 Milano, Italy; (1) orcid.org/0000-0003-3792-3661

Sergio Brovelli - Dipartimento di Scienza dei Materiali, Università degli Studi di Milano Bicocca, I-20125 Milano, Italy; (1) orcid.org/0000-0002-5993-855X

Sara Bals - Electron Microscopy for Materials Science (EMAT), University of Antwerp, B-2020 Antwerp, Belgium; 이이.org/ 0000-0002-4249-8017

Marc-Etienne Moret - Organic Chemistry and Catalysis, Debye Institute for Nanomaterials Science, Utrecht University, 3584

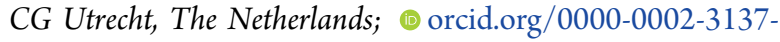
6073

Complete contact information is available at:

https://pubs.acs.org/10.1021/acsnano.0c04744

\section{Notes}

The authors declare no competing financial interest.

\section{ACKNOWLEDGMENTS}

S.B. and C.d.M.D. acknowledge financial support from the division of Chemical Sciences (CW) of The Netherlands Organization for Scientific Research (NWO) under Grant No. TOP.715.016.001. W.A. acknowledges an Individual Fellowship from the Marie Sklodowska-Curie actions (MSCA) under the EU's Horizon 2020 program (Grant No. 797153, SOPMEN). This project has received funding from the European Commission Grant (EUSMI E180900184) and European Research Council (ERC Consolidator Grant No. 815128 - REALNANO).

\section{REFERENCES}

(1) Goldbery, Y. A. Handbook Series on Semiconductor Parameters; Levinshtein, M., Rumyantsev, S., Shur, M., Eds.; World Scientific: London, 1996; Vol.1, pp 191-213.

(2) Mohammad, A.; Das, S. R.; Khan, M. R.; Alam, M. A.; Janes, D. B. Wavelength-Dependent Absorption in Structurally Tailored Randomly Branched Vertical Arrays of InSb Nanowires. Nano Lett. 2012, 12, 6112-6118.

(3) Nilsson, H. A.; Caroff, P.; Thelander, C.; Lind, E.; Karlström, O.; Wernersson, L. E. Temperature Dependent Properties of InSb and InAs Nanowire Field-Effect Transistors. Appl. Phys. Lett. 2010, 96, 153505

(4) Snyder, G. J.; Toberer, E. S. Complex Thermoelectric Materials. Nat. Mater. 2008, 7, 105-114.

(5) Isaacson, R. A. Electron Spin Resonance in $n$-Type InSb. Phys. Rev. 1968, 169, 312-314.

(6) Warburton, R. J. Single Spins in Self-Assembled Quantum Dots. Nat. Mater. 2013, 12, 483-493. 
(7) van den Berg, J. W. G.; Nadj-Perge, S.; Pribiag, V. S.; Plissard, S. R.; Bakkers, E. P. A. M.; Frolov, S. M.; Kouwenhoven, L. P. Fast SpinOrbit Qubit in an Indium Antimonide Nanowire. Phys. Rev. Lett. 2013, 110, 066806.

(8) Stern, A.; Lindner, N. H. Topological Quantum ComputationFrom Basic Concepts to First Experiments. Science 2013, 339, 11791184.

(9) Zhang, H.; Liu, C.; Gazibegovic, S.; Xu, D.; Logan, J. A.; Wang, G.; van Loo, N.; Bommer, J. D. S.; de Moor, M. W. A.; Car, D.; Op het Veld, R. L. M.; van Veldhoven, P. J.; Koelling, S.; Verheijen, M. A.; Pendharkar, M.; Pennachio, D. J.; Shojaei, B.; Lee, J. S.; Palmstrøm, C. J.; Bakkers, E. P. A. M.; et al. Quantized Majorana Conductance. Nature 2018, 556, 74-79.

(10) Sestoft, J. E.; Kanne, T.; Gejl, A. N.; von Soosten, M.; Yodh, J. S.; Sherman, D.; Tarasinski, B.; Wimmer, M.; Johnson, E.; Deng, M.; Nygård, J.; Jespersen, T. S.; Marcus, C. M.; Krogstrup, P. Engineering Hybrid Epitaxial InAsSb/Al Nanowires for Stronger Topological Protection. Phys. Rev. Mater. 2018, 2, 044202.

(11) Lutchyn, R. M.; Bakkers, E. P. A. M.; Kouwenhoven, L. P.; Krogstrup, P.; Marcus, C. M.; Oreg, Y. Majorana Zero Modes in Superconductor-Semiconductor Heterostructures. Nat. Rev. Mater. 2018, 3, 52-68.

(12) Kagan, C. R.; Lifshitz, E.; Sargent, E. H.; Talapin, D. V. Building Devices from Colloidal Quantum Dots. Science 2016, 353, No. aac5523.

(13) Tang, X.; Ackerman, M. M.; Guyot-Sionnest, P. Acquisition of Hyperspectral Data with Colloidal Quantum Dots. Laser Photonics Rev. 2019, 13, 1900165.

(14) Hemmer, E.; Benayas, A.; Légaré, F.; Vetrone, F. Exploiting the Biological Windows: Current Perspectives on Fluorescent Bioprobes Emitting above 1000 nm. Nanoscale Horiz. 2016, 1, 168-184.

(15) Xu, J.; Voznyy, O.; Liu, M.; Kirmani, A. R.; Walters, G.; Munir, R.; Abdelsamie, M.; Proppe, A. H.; Sarkar, A.; García de Arquer, F. P.; Wei, M.; Sun, B.; Liu, M.; Ouellette, O.; Quintero-Bermudez, R.; Li, J.; Fan, J.; Quan, L.; Todorovic, P.; Tan, H.; et al. 2D Matrix Engineering for Homogeneous Quantum Dot Coupling in Photovoltaic Solids. Nat. Nanotechnol. 2018, 13, 456-462.

(16) Sills, A.; Harrison, P.; Califano, M. Exciton Dynamics in InSb Colloidal Quantum Dots. J. Phys. Chem. Lett. 2016, 7, 31-35.

(17) Tadjine, A.; Niquet, Y.; Delerue, C. Universal Behavior of Electron g-Factors in Semiconductor Nanostructures. Phys. Rev. B: Condens. Matter Mater. Phys. 2017, 95, 235437.

(18) Witek, B. J.; Heeres, R. W.; Perinetti, U.; Bakkers, E. P. A. M.; Kouwenhoven, L. P.; Zwiller, V. Measurement of the g-Factor Tensor in a Quantum Dot and Disentanglement of Exciton Spins. Phys. Rev. B: Condens. Matter Mater. Phys. 2011, 84, 195305.

(19) Barrigoń, E.; Heurlin, M.; Bi, Z.; Monemar, B.; Samuelson, L. Synthesis and Applications of III-V Nanowires. Chem. Rev. 2019, 119, 9170-9220.

(20) Lin, A.; Shapiro, J. N.; Eisele, H.; Huffaker, D. L. Tuning the $\mathrm{Au}-F r e e$ InSb Nanocrystal Morphologies Grown by Patterned MetalOrganic Chemical Vapor Deposition. Adv. Funct. Mater. 2014, 24, 4311-4316.

(21) Badawy, G.; Gazibegovic, S.; Borsoi, F.; Heedt, S.; Wang, C.; Koelling, S.; Verheijen, M. A.; Kouwenhoven, L. P.; Bakkers, E. P. A. M. High Mobility Stemless InSb Nanowires. Nano Lett. 2019, 19, $3575-3582$.

(22) Liu, W.; Chang, A. Y.; Schaller, R. D.; Talapin, D. V. Colloidal InSb Nanocrystals. J. Am. Chem. Soc. 2012, 134, 20258-20261.

(23) Yarema, M.; Kovalenko, M. V. Colloidal Synthesis of InSb Nanocrystals with Controlled Polymorphism Using Indium and Antimony Amides. Chem. Mater. 2013, 25, 1788-1792.

(24) Zhao, T.; Oh, N.; Jishkariani, D.; Zhang, M.; Wang, H.; Li, N.; Lee, J. D.; Zeng, C.; Muduli, M.; Choi, H.; Su, D.; Murray, C. B.; Kagan, C. R. General Synthetic Route to High-Quality Colloidal III-V Semiconductor Quantum Dots Based on Pnictogen Chlorides. J. Am. Chem. Soc. 2019, 141, 15145-15152.
(25) Evans, C. M.; Castro, S. L.; Worman, J. J.; Raffaelle, R. P. Synthesis and Use of Tris(trimethylsilyl)antimony for the Preparation of InSb Quantum Dots. Chem. Mater. 2008, 20, 5727-5730.

(26) Maurice, A.; Lopez Haro, M.; Hyot, B.; Reiss, P. Synthesis of Colloidal Indium Antimonide Nanocrystals Using Stibine. Part. Part. Syst. Charact. 2013, 30, 828-831.

(27) Crisp, R. W.; Grimaldi, G.; De Trizio, L.; Evers, W. H.; Kirkwood, N.; Kinge, S.; Manna, L.; Siebbeles, L. D. A.; Houtepen, A. J. Selective Antimony Reduction Initiating the Nucleation and Growth of InSb Quantum Dots. Nanoscale 2018, 10, 11110-11116.

(28) Tamang, S.; Kim, K.; Choi, H.; Kima, Y.; Jeong, S. Synthesis of Colloidal InSb Nanocrystals via in Situ Activation of $\mathrm{InCl}_{3}$. Dalton Trans. 2015, 44, 16923-16928.

(29) Schulz, S. The Chemistry of Group 13/15 Compounds (III-V Compounds) with the Higher Homologues of Group 15, Sb and Bi. Coord. Chem. Rev. 2001, 215, 1-37.

(30) Edwards, A. J.; Paver, M. A.; Rennie, M.-A.; Raithby, P. R.; Russel, C. A.; Wright, D. S. Intramolecular versus Intermolecular Bonding in Amidoantimony Dimers; Syntheses and Structures of $\left\{\mathrm{Sb}\left(\mathrm{NMe}_{2}\right)[\mu-\mathrm{N}(\mathrm{mpy})]\right\}_{2}$ (mpy = 4-Methylpyridin-2-yl) and [Sb$\left.\left(\mathrm{NMe}_{2}\right)\left\{\mu-\mathrm{N}\left[\mathrm{C}_{6} \mathrm{H}_{2}(\mathrm{OMe})_{3}-3,4,5\right]\right\}\right]_{2}$. J. Chem. Soc., Dalton Trans. 1994, 2963-2966.

(31) Harmgarth, N.; Liebing, P.; Zorner, F.; Silinskas, M.; Burte, E. P.; Edelmann, F. T. Synthesis and Crystal Structures of the First Antimony(III) Aziridinides. Inorg. Chem. 2017, 56, 4267-4270.

(32) Eisler, D. J.; Chivers, T. Bis(1-Amino)cyclodistib(III)azanes: The First Structural Characterization of Cis and Trans Isomers of a Single Cyclodipnict(III)azane. Inorg. Chem. 2006, 45, 10734-10742.

(33) Beswick, M. A.; Davies, M. K.; Paver, M. A.; Raithby, P. R.; Steiner, A.; Wright, D. S. $\left[\mathrm{Sb}_{12}\left\{(2-\mathrm{MeO}) \mathrm{C}_{6} \mathrm{H}_{4} \mathrm{~N}\right\}_{18}\right] \cdot 6$ THF; A Twenty-Four Membered Imidoantimony(III) Metallacycle. Angew. Chem., Int. Ed. Engl. 1996, 35, 1508-1510.

(34) Bryant, R.; James, S. C.; Jeffery, J. C.; Norman, N. C.; Orpen, A. G.; Weckenmann, U. Antimony Imido and Imido-Amido Compounds: A New Route to an Imidoantimony Macrocycle. J. Chem. Soc., Dalton Trans. 2000, 4007-4009.

(35) Weiss, J.; Eisenhuth, W. Die Kristall- und Molekülstruktur von $\mathrm{As}_{4}\left(\mathrm{NCH}_{3}\right)_{6}$. Z. Anorg. Allg. Chem. 1967, 350, 9-17.

(36) Buffard, A.; Dreyfuss, S.; Nadal, B.; Heuclin, H.; Xu, X.; Patriarche, G.; Mézailles, N.; Dubertret, B. Mechanistic Insight and Optimization of InP Nanocrystals Synthesized with Aminophosphines. Chem. Mater. 2016, 28, 5925-5934.

(37) Baldwin, R. A.; Foos, E. E.; Wells, R. L.; White, P. S.; Rheingold, A. L.; Yap, G. P. A. Synthesis and Characterization of Potential Single-Source Precursors to Group 13 - Antimonides. Organometallics 1996, 15, 5035-5038.

(38) Foos, E. E.; Wells, R. L.; Rheingold, A. L. Preparation of Nanocrystalline Indium Antimonide through t-Hydride Elimination from New Indium-Antimony Single-Source Precursors. J. Cluster Sci. 1999, 10, 121.

(39) He, M.; Protesescu, L.; Caputo, R.; Krumeich, F.; Kovalenko, M. V. A General Synthesis Strategy for Monodisperse Metallic and Metalloid Nanoparticles (In, Ga, Bi, Sb, Zn, Cu, Sn and Their Alloys) via in Situ Formed Metal Long-Chain Amides. Chem. Mater. 2015, 27, 635-647.

(40) Donega, C.; de, M. Synthesis and Properties of Colloidal Heteronanocrystals. Chem. Soc. Rev. 2011, 40, 1512-1546.

(41) García-Rodríguez, R.; Hendricks, M. P.; Cossairt, B. M.; Liu, H.; Owen, J. S. Conversion Reactions of Cadmium Chalcogenide Nanocrystal Precursors. Chem. Mater. 2013, 25, 1233-1249.

(42) Sowers, K. L.; Swartz, B.; Krauss, T. D. Chemical Mechanisms of Semiconductor Nanocrystal Synthesis. Chem. Mater. 2013, 25, $1351-1362$.

(43) Cossairt, B. M. Shining Light on Indium Phosphide Quantum Dots: Understanding the Interplay among Precursor Conversion, Nucleation, and Growth. Chem. Mater. 2016, 28, 7181-7189.

(44) Van der Stam, W.; Berends, A. C.; de Mello Donegá, C. Prospects of Colloidal Copper Chalcogenide Nanocrystals. ChemPhysChem 2016, 17, 559-581. 
(45) Van Hyning, D. L.; Klemperer, W. G.; Zukoski, C. F. Silver Nanoparticle Formation: Predictions and Verification of the Aggregative Growth Model. Langmuir 2001, 17, 3128-3135.

(46) Polte, J.; Erler, R.; Thunemann, A. F.; Sokolov, S.; Ahner, T. T.; Rademann, K.; Emmerling, F.; Kraehnert, R. Nucleation and Growth of Gold Nanoparticles Studied via in Situ Small Angle X-Ray Scattering at Millisecond Time Resolution. ACS Nano 2010, 4, 10761082.

(47) Polte, J.; Tuaev, X.; Wuithschick, M.; Fischer, A.; Thuenemann, A. F.; Rademann, K.; Kraehnert, R.; Emmerling, F. Formation Mechanism of Colloidal Silver Nanoparticles: Analogies and Differences to the Growth of Gold Nanoparticles. ACS Nano 2012, 6, 5791-5802.

(48) Zheng, H.; Smith, R. K.; Jun, Y. W.; Kisielowski, C.; Dahmen, U.; Alivisatos, A. P. Observation of Single Colloidal Platinum Nanocrystal Growth Trajectories. Science 2009, 324, 1309-1312.

(49) Yuk, J. M.; Park, J.; Ercius, P.; Kim, K.; Hellebusch, D. J.; Crommie, M. F.; Lee, J. Y.; Zettl, A.; Alivisatos, A. P. High-Resolution EM of Colloidal Nanocrystal Growth Using Graphene Liquid Cells. Science 2012, 336, 61-64.

(50) van der Stam, W.; Gradmann, S.; Altantzis, T.; Ke, X.; Baldus, M.; Bals, S.; de Mello Donega, C. Shape Control of Colloidal $\mathrm{Cu}_{2-\mathrm{x}} \mathrm{S}$ Polyhedral Nanocrystals by Tuning the Nucleation Rates. Chem. Mater. 2016, 28, 6705-6715.

(51) Lee, J.; Yang, J.; Kwon, S. G.; Hyeon, T. Nonclassical Nucleation and Growth of Inorganic Nanoparticles. Nat. Rev. Mater. 2016, 1, 16034.

(52) Wang, F.; Richards, V. N.; Shields, S. P.; Buhro, W. E. Kinetics and Mechanisms of Aggregative Nanocrystal Growth. Chem. Mater. 2014, 26, 5-21.

(53) Privman, V.; Goia, D. V.; Park, J.; Matijevic, E. J. Mechanism of Formation of Monodispersed Colloids by Aggregation of Nanosize Precursors. J. Colloid Interface Sci. 1999, 213, 36-45.

(54) Dixit, N. M.; Zukoski, C. F. Nucleation Rates and Induction Times during Colloidal Crystallization: Links between Models and Experiments. Phys. Rev. E: Stat. Phys., Plasmas, Fluids, Relat. Interdiscip. Top. 2002, 66, 051602.

(55) Pasumansky, L.; Goralski, C. T.; Singaram, B. Lithium Aminoborohydrides: Powerful, Selective, Air-Stable Reducing Agents. Org. Process Res. Dev. 2006, 10, 959-970.

(56) Qian, Y.; Yang, Q. Straight Indium Antimonide Nanowires with Twinning Superlattices via a Solution Route. Nano Lett. 2017, 17, $7183-7190$.

(57) Grodzinska, D.; Evers, W. H.; Dorland, R.; van Rijssel, J.; van Huis, M.; Meijerink, A.; de Mello Donegá, C.; Vanmaekelbergh, D. Two-Fold Emission from the S-Shell of $\mathrm{PbSe}($ Core $) / \mathrm{CdSe}($ Shell) Quantum Dots. Small 2011, 7, 3493-3501.

(58) Efros, Al. L.; Rosen, M. Quantum Size Level Structure of Narrow-Gap Semiconductor Nanocrystals: Effect of Band Coupling. Phys. Rev. B: Condens. Matter Mater. Phys. 1998, 58, 7120-7135.

(59) Sukkabot, W. Tight-Binding Theory of the Excitonic States in Colloidal InSb Nanostructures. Mater. Sci. Semicond. Process. 2014, 27, $51-55$.

(60) Kuçur, E.; Boldt, F. M.; Cavaliere-Jaricot, S.; Ziegler, J.; Nann, T. Quantitative Analysis of Cadmium Selenide Nanocrystal Concentration by Comparative Techniques. Anal. Chem. 2007, 79, 8987-8993.

(61) Cademartiri, L.; Montanari, E.; Calestani, G.; Migliori, A.; Guagliardi, A.; Ozin, G. A. Size-Dependent Extinction Coefficients of PbS Quantum Dots. J. Am. Chem. Soc. 2006, 128, 10337-10346.

(62) Moreels, I.; Lambert, K.; De Muynck, D.; Vanhaecke, F.; Poelman, D.; Martins, J. C.; Allan, G.; Hens, Z. Composition and Size-Dependent Extinction Coefficient of Colloidal PbSe Quantum Dots. Chem. Mater. 2007, 19, 6101-6106.

(63) de Mello Donega, C.; Koole, R. Size Dependence of the Spontaneous Emission Rate and Absorption Cross Section of CdSe and CdTe Quantum Dots. J. Phys. Chem. C 2009, 113, 6511-6520.
(64) Jasieniak, J.; Smith, L.; van Embden, J.; Mulvaney, P.; Califano, M. Re-Examination of the Size-Dependent Absorption Properties of CdSe Quantum Dots. J. Phys. Chem. C 2009, 113, 19468-19474.

(65) Xia, C.; Wu, W.; Yu, T.; Xie, X.; van Oversteeg, C.; Gerritsen, H. C.; de Mello Donega, C. Size-Dependent Band-Gap and Molar Absorption Coefficients of Colloidal $\mathrm{CuInS}_{2}$ Quantum Dots. ACS Nano 2018, 12, 8350-8361.

(66) Banin, U.; Lee, C. J.; Guzelian, A. A.; Kadavanich, A. V.; Alivisatos, A. P.; Jaskolski, W.; Bryant, G. W.; Efros, Al. L.; Rosen, M. Size-Dependent Electronic Level Structure of InAs Nanocrystal Quantum Dots: Test of Multiband Effective Mass Theory. J. Chem. Phys. 1998, 109, 2306-2309.

(67) Williamson, A. J.; Franceschetti, A.; Fu, H.; Wang, L. W.; Zunger, A. Indirect Band Gaps in Quantum Dots Made from DirectGap Bulk Materials. J. Electron. Mater. 1999, 28, 414-425.

(68) Chang, A. Y.; Liu, W.; Talapin, D. V.; Schaller, R. D. Carrier Dynamics in Highly Quantum-Confined Colloidal Indium Antimonide Nanocrystals. ACS Nano 2014, 8, 8513-8519.

(69) Wang, T.; Vaxenburg, R.; Liu, W.; Rupich, S. M.; Lifshitz, E.; Efros, A. L.; Talapin, D. V.; Sibener, S. J. Size-Dependent Energy Levels of InSb Quantum Dots Measured by Scanning Tunneling Spectroscopy. ACS Nano 2015, 9, 725-732.

(70) Allan, G.; Delerue, C. Confinement Effects in PbSe Quantum Wells and Nanocrystals. Phys. Rev. B: Condens. Matter Mater. Phys. 2004, 70, 245321.

(71) Moreels, I.; Lambert, K.; Smeets, D.; De Muynck, D.; Nollet, T.; Martins, J. C.; Vanhaecke, F.; Vantomme, A.; Delerue, C.; Allan, G.; Hens, Z. Size-Dependent Optical Properties of Colloidal PbS Quantum Dots. ACS Nano 2009, 3, 3023-3030.

(72) Narayanaswamy, A.; Feiner, L. F.; Meijerink, A.; van der Zaag, P. J. The Effect of Temperature and Dot Size on the Spectral Properties of Colloidal InP/ZnS Core-Shell Quantum Dots. ACS Nano 2009, 3, 2539-2546.

(73) Cho, E.; Jang, H.; Lee, J.; Jang, E. Modeling on the Size Dependent Properties of InP Quantum Dots: A Hybrid Functional Study. Nanotechnology 2013, 24, 215201.

(74) Zemel, J. N.; Jensen, J. D.; Schoolar, R. B. Electrical and Optical Properties of Epitaxial Films of PbS, PbSe, PbTe, and SnTe. Phys. Rev. 1965, 140, A330-342.

(75) Hilsum, C.; Fray, S.; Smith, C. The Optical Frequencies and Dielectric Constants of InP. Solid State Commun. 1969, 7, 10571059. 